\title{
New Design Methods for Two-Dimensional Filters Based on 1D Prototypes and Spectral Transformations
}

\author{
Radu Matei \\ Technical University "Gh.Asachi" of Iasi \\ Romania
}

\section{Introduction}

The field of two-dimensional filters and their design methods have been approached by many researchers, for more than three decades (Lim, 1990; Lu \& Antoniou, 1992). A commonly-used design technique for 2D filters is to start from a specified 1D prototype filter and transform its transfer function using various frequency mappings in order to obtain a 2D filter with a desired frequency response. These are essentially spectral transformations from $s$ to $z$ plane via bilinear or Euler transformations followed by $\mathrm{z}$ to $\left(z_{1}, z_{2}\right)$ mappings, approached in early reference papers (Pendergrass et al., 1976; Hirano \& Aggarwal, 1978; Harn \& Shenoi, 1986). Generally these spectral transformations conserve stability, so from 1D prototypes various stable recursive 2D filters can be obtained.

There are several classes of filters with orientation-selective frequency response, useful in some image processing tasks, such as edge detection, motion analysis etc. An important class are the steerable filters, synthesized as a linear combination of a set of basis filters (Freeman \& Adelson, 1991). Another important category are Gabor filters, with applications in some complex tasks in image processing. A major reference on oriented filters is (Chang \& Aggarwal, 1977), where a technique for rotating the frequency response of separable filters is developed. The proposed method considers transfer functions in rational powers of $z$ and realized by input-output signal array interpolations. Anisotropic, in particular elliptically-shaped filters have also been studied extensively and are used in some interesting applications, e.g. in remote sensing for directional smoothing applied to weather images (Lakshmanan, 2004), also in texture segmentation and pattern recognition. Other directionally selective operators are proposed in (Danielsson, 1980).

Another particular class are the wedge filters, named so due to their symmetric wedge-like shape in the frequency plane. They find interesting applications, e.g. in texture classification (Randen \& Husoy, 1999). In (Simoncelli \& Farid, 1996) the steerable wedge filters were introduced, which are used to analyze local orientation patterns in images.

Linear filter banks of various shapes, combined with pattern recognition techniques have been widely used in image analysis and enhancement, texture segmentation etc. In particular, directional filter banks provide an orientation-selective image decomposition. 
The Bamberger directional filter bank (Bamberger \& Smith, 1992), is a purely directional decomposition that provides excellent frequency domain selectivity with low computational complexity. This family of filter banks has been successfully used for image denoising, character recognition, image enhancement etc. Diamond filters are currently used as antialiasing filters for the conversion between signals sampled on the rectangular sampling grid and the quincunx sampling grid. Some design techniques, mainly for FIR diamond filters were developed (Lim \& Low, 1997; Low \& Lim, 1998).

Stability of the two-dimensional recursive filters is also an important issue and is more complicated than for 1D filters. For 2D filters, in general, it is quite difficult to take stability constraints into account during the stage of approximation (O'Connor, 1978). For this reason, various techniques were developed to separate the stability from the approximation problem. If the designed filter becomes unstable, some stabilization procedures are needed (Jury, 1977). Unlike 1D filters, in 2D filters the numerator can affect the filter stability and can sometimes stabilize an otherwise unstable filter.

The design methods in the frequency domain described in this chapter are also based on spectral transformations, or frequency transformations, a term more often used in text. Starting from an 1D prototype filter with a desired characteristics, for instance low-pass maximally-flat, selective low-pass or band-pass etc., some specific spectral transformations will be applied in order to obtain the 2D filter with a desired shape. Various types of 2D filters will be approached: directional selective filters, oriented wedge filters, fan filters, diamond-shaped filters etc. All these filters have already found specific applications in image processing. The general case will be approached, when we start from a 1D prototype which is a common digital filter, either maximally-flat or equiripple (Butterworth, Chebyshev, elliptic etc.) given by a transfer function in variable $z$, which is decomposed into a product of elementary functions of first or second order. In this case the design consists in finding the specific complex frequency transformation from the variable $\mathrm{z}$ to the complex plane $\left(z_{1}, z_{2}\right)$. Once found this mapping, the $2 \mathrm{D}$ filter function results directly through substitution. The case of zero-phase $2 \mathrm{D}$ filters will be treated as well, since they are very useful in various image filtering applications due to the absence of phase distortions. This method is at the same time simple, efficient and versatile, since once found the adequate frequency transformation, it can be applied to different prototype filters obtaining the 2D filter. The latter inherits the selectivity properties of its 1D counterpart (bandwidth, flatness, transition band etc.). Changing the prototype filter parameters will change the properties of the obtained 2D filter. All the proposed design techniques are mainly analytical but also involve numerical optimization, in particular rational approximations (Padé or Chebyshev-Padé). Since the design starts from a factorized transfer function, the 2D filter function will also result directly factorized, which is a major advantage in its implementation. For each specified shape of the 2D filter, a particular frequency transformation is derived.

Some proposed methods involve the bilinear transform as an intermediate step. Depending on their shape, the designed filters may present non-linearity distortions towards the margins of the frequency plane, due to the frequency warping effect. In order to compensate for these errors, a pre-warping may be applied, which increases the filter order. Other proposed methods avoid from the start the use of bilinear transform and the filter coefficients result through a change of frequency variable and a bivariate Taylor or 
Chebyshev expansion of the filter frequency response. Finally the filter transfer function in $z_{1}$ and $z_{2}$ results directly by identification of the $2 \mathrm{D} Z$ transform terms.

An original design method is proposed in section 5 for a class of filters specified by a periodic function expressed in polar coordinates in the frequency plane. The contour plots of their frequency response, resulted as sections with planes parallel with the frequency plane, can be defined as closed curves, described in terms of a variable radius which can be written as a rational and periodic function of the current angle formed with one of the axes. In this class of filters we studied two-lobe filters, selective four-lobe filters with an arbitrary orientation angle, fan filters and diamond filters.

Several related design methods proposed by the author for other types of 2D zero-phase filters, especially with circular and elliptical symmetry were developed in (Matei, 2009, b). In the last section of the chapter, a few applications of the designed wedge filter will be presented through simulation results.

\section{1D Prototype Filters and Spectral Transformations Used in 2D Filter Design}

An essential step in designing temporal and spatial filters is the approximation. As mentioned in the above introduction, the proposed design methods for $2 \mathrm{D}$ recursive filters are based on 1D prototype filters with imposed specifications. For the 2D filters approached here, we start from 1D digital filters described by a transfer function $\mathrm{H}(\mathrm{z})$, resulted from one of the common approximations (Butterworth, Chebyshev, elliptical etc.) and satisfying the desired specifications. Analog prototype filters with transfer functions in variable s can also be used. The choice depends on the 2D filter type, which requires a specific frequency transformation; this must be as simple as possible in order to obtain an efficient, low-order filter. On the other hand we may start from a complex or real-valued filter prototype. In the latter case zero-phase 2D filters will result, which are free of phase distortions.

Let us consider a recursive digital filter of order $\mathrm{N}$ with the transfer function:

$$
H(z)=\frac{P(z)}{Q(z)}=\sum_{i=0}^{M} p_{i} \cdot z^{i} / \sum_{j=0}^{N} q_{j} \cdot z^{j}
$$

We consider this general transfer function with $\mathrm{M}=\mathrm{N}$ factorized into rational functions of first and second order. An odd order filter $\mathrm{H}(\mathrm{z})$ has at least one first order factor:

$$
\mathrm{H}_{1}(\mathrm{z})=\left(\mathrm{b}_{1} \mathrm{z}+\mathrm{b}_{0}\right) /\left(\mathrm{z}+\mathrm{a}_{0}\right)
$$

The transfer function also contains second-order factors referred to as biquad functions:

$$
\mathrm{H}_{2}(\mathrm{z})=\left(\mathrm{b}_{2} \mathrm{z}^{2}+\mathrm{b}_{1} \mathrm{z}+\mathrm{b}_{0}\right) /\left(\mathrm{z}^{2}+\mathrm{a}_{1} \mathrm{z}+\mathrm{a}_{0}\right)
$$

where in general the second-order polynomials at the numerator and denominator have complex-conjugated roots. The main issue approached in this chapter is to find the transfer function of the desired $2 \mathrm{D}$ filter $\mathrm{H}_{2 \mathrm{D}}\left(\mathrm{z}_{1}, \mathrm{z}_{2}\right)$ using appropriate frequency transformations of 
the form: $\omega \rightarrow \mathrm{F}\left(\omega_{1}, \omega_{2}\right)$. The elementary transfer functions (2) and (3) can be put into the form of a complex frequency response:

$$
\begin{gathered}
H_{1}(j \omega)=\left(b_{0}+b_{1} \cos \omega+j b_{1} \sin \omega\right) /\left(a_{0}+\cos \omega+j \sin \omega\right) \\
H_{2}(j \omega)=\frac{b_{1}+\left(b_{2}+b_{0}\right) \cos \omega+j\left(b_{2}-b_{0}\right) \sin \omega}{a_{1}+\left(1+a_{0}\right) \cos \omega+j\left(1-a_{0}\right) \sin \omega}=\frac{P(\omega)}{Q(\omega)}
\end{gathered}
$$

We notice that the first- and second-order functions have a similar form when expressed as a ratio of complex numbers. Therefore, as shown further, the corresponding $2 \mathrm{D}$ transfer functions will be implemented with convolution kernels of the same size. The next step starts from the expressions (4) and (5) of the frequency response and uses of the following accurate rational approximations for sine and cosine on $[-\pi, \pi]$ :

$$
\begin{aligned}
& \cos \omega \cong \frac{1-0.435949 \cdot \omega^{2}+0.011319 \cdot \omega^{4}}{1+0.06095 \cdot \omega^{2}+0.0037557 \cdot \omega^{4}}=\frac{C(\omega)}{Q(\omega)} \\
& \sin \omega \cong \frac{\omega \cdot\left(1-0.101046 \cdot \omega^{2}\right)}{1+0.06095 \cdot \omega^{2}+0.0037557 \cdot \omega^{4}}=\frac{S(\omega)}{Q(\omega)}
\end{aligned}
$$

The above expressions were obtained through a Chebyshev-Padé approximation, found using a symbolic computation software. The advantage of these expressions is that they have the same denominator and can be directly substituted into (4) and (5), yielding a rational expression of the frequency response $\mathrm{H}\left(\mathrm{e}^{\mathrm{j} \omega}\right)$ of the same order.

In order to design a zero-phase 2D filter, we start from zero-phase prototypes, with realvalued transfer functions. Such a filter may be obtained by finding a rational approximation of the magnitude characteristics of the given prototype. The magnitude $|H(\omega)|$ taken from $\mathrm{H}(\mathrm{z})=\mathrm{H}\left(\mathrm{e}^{\mathrm{j} \omega}\right)$ of the general form (1) can be approximated by a ratio of polynomials in even powers of frequency $\omega$, on the range $\omega \in[-\pi, \pi]$. In general this filter will be described by:

$$
\mathrm{H}_{\mathrm{p}}(\omega)=\sum_{\mathrm{j}=0}^{\mathrm{M}} \mathrm{b}_{\mathrm{j}} \cdot \omega^{2 \mathrm{j}} / \sum_{\mathrm{k}=0}^{\mathrm{N}} \mathrm{a}_{\mathrm{k}} \cdot \omega^{2 \mathrm{k}}
$$

where $\mathrm{M} \leq \mathrm{N}$ and $\mathrm{N}$ is the filter order. In (Matei, 2009, b) a different version of approximation was proposed, which using the change of variable $\omega=\arccos x \Leftrightarrow x=\cos \omega$ yields a rational approximation of $|\mathrm{H}(\omega)|$ in the variable $\cos \omega$ on the range $\omega \in[-\pi, \pi]$ :

$$
|H(\omega)| \cong \sum_{n=1}^{N} b_{n} \cos ^{n} \omega / \sum_{m=1}^{N} a_{m} \cos ^{m} \omega
$$

This rational trigonometric approximation is particularly useful in designing zero-phase circular or elliptically-shaped filters, approached in (Matei, 2009, b), but less efficient for other 2D filters like directional, wedge-shaped etc. 
For instance, considering as $1 \mathrm{D}$ prototype a type-2 Chebyshev digital filter with the parameters: order $\mathrm{N}=4$, stopband attenuation $\mathrm{R}_{\mathrm{s}}=40 \mathrm{~dB}$ and passband-edge frequency $\omega_{\mathrm{p}}=0.5$, where 1.0 is half the sampling frequency, its transfer function in $\mathrm{z}$ has the form:

$$
H(z)=\left(0.012277 \cdot z^{2}-0.012525 \cdot z+0.012277\right) /\left(z^{2}-1.850147 \cdot z+0.862316\right)
$$

Using a Chebyshev-Padé approximation we can determine the following real-valued zerophase frequency response which approximates accurately the magnitude of the function (10):

$$
\left|\mathrm{H}\left(\mathrm{e}^{\mathrm{j} \omega}\right)\right| \cong \mathrm{H}_{\mathrm{a} 1}(\omega)=\left(0.9403-0.57565 \cdot \omega^{2}+0.0947 \cdot \omega^{4}\right) /\left(1-2.067753 \cdot \omega^{2}+4.663147 \cdot \omega^{4}\right)
$$

\section{Directional Filters}

We propose a design method for a class of 2D oriented low-pass filters which select narrow domains along specified directions in the frequency plane $\left(\omega_{1}, \omega_{2}\right)$. Such filters can be used in selecting lines with a given orientation from an input image. Since we envisage to design filters of minimum order, we use IIR filters as prototypes. Here we treat the general case using a complex frequency transformation. Other related methods for directional filter design were discussed in (Matei, 2009, b).

Starting from a real-valued prototype $\mathrm{H}\left(\omega_{1}\right)$, a $2 \mathrm{D}$ oriented filter is obtained by rotating the axes of the plane $\left(\omega_{1}, \omega_{2}\right)$ with an angle $\varphi$, as described by the linear transformation:

$$
\left[\begin{array}{l}
\omega_{1} \\
\omega_{2}
\end{array}\right]=\left[\begin{array}{cc}
\cos \varphi & \sin \varphi \\
-\sin \varphi & \cos \varphi
\end{array}\right] \cdot\left[\begin{array}{l}
\bar{\omega}_{1} \\
\bar{\omega}_{2}
\end{array}\right]
$$

where $\omega_{1}, \omega_{2}$ are the original frequency variables and $\bar{\omega}_{1}, \bar{\omega}_{2}$ the rotated ones. The filter orientation is specified by an angle $\varphi$ about $\omega_{1}$-axis and is defined by the following 1D to 2D spectral transformation of the frequency response $H\left(\omega_{1}, \omega_{2}\right): \omega \rightarrow \omega_{1} \cos \varphi+\omega_{2} \sin \varphi$. By substitution, we obtain the transfer function of the oriented filter $\mathrm{H}_{\varphi}\left(\omega_{1}, \omega_{2}\right)$ :

$$
\mathrm{H}_{\varphi}\left(\omega_{1}, \omega_{2}\right)=\mathrm{H}\left(\omega_{1} \cos \varphi+\omega_{2} \sin \varphi\right)
$$

The filter $H_{\varphi}\left(\omega_{1}, \omega_{2}\right)$ has the magnitude along the line $\omega_{1} \cos \varphi+\omega_{2} \sin \varphi=0$ identical with the prototype $H(\omega)$ and constant along the line $\omega_{1} \sin \varphi-\omega_{2} \cos \varphi=0$ (longitudinal axis). Next we will determine a convenient $1 \mathrm{D}$ to $2 \mathrm{D}$ complex transformation which allows for obtaining an oriented 2D filter from a 1D prototype filter. The special case of zero-phase directional filters was extensively treated in (Matei, 2009, b).

\subsection{Design Method for 2D Directional Filters Based on Frequency Transformation}

In the following section we will introduce a design method which allows one to obtain a 2D discrete orientation-selective filter. The desired filter will be derived directly from a 1D discrete prototype filter through a complex frequency transformation. 
A discrete 1D filter is generally described by a transfer function $H(z)$. The complex variable $\mathrm{z}=\mathrm{e}^{\mathrm{j} \omega}=\mathrm{e}^{\mathrm{s}}$ will be mapped into a 2D function $\mathrm{F}_{\varphi}\left(\mathrm{z}_{1}, \mathrm{z}_{2}\right)$, where the index $\varphi$ denotes the dependence upon the orientation angle. Using the frequency transformation (13) which defines the orientation-selective filter with the orientation angle $\varphi$, we have successively:

$$
e^{j\left(\omega_{1} \cos \varphi+\omega_{2} \sin \varphi\right)}=e^{s_{1} \cos \varphi} \cdot e^{s_{2} \sin \varphi}=\left(z_{1}\right)^{\cos \varphi} \cdot\left(z_{2}\right)^{\sin \varphi}=f_{1}\left(s_{1}\right) \cdot f_{2}\left(s_{2}\right)
$$

Therefore the complex frequency transformation is $\mathrm{z} \rightarrow \mathrm{z}_{1}^{\cos \varphi} \cdot \mathrm{z}_{2}^{\sin \varphi}$. In (Chang \& Aggarwal, 1977) the frequency transformation used is $z \rightarrow z_{1} \cdot z_{2}^{\beta / \alpha}$, where $\alpha$ and $\beta$ are integers. The rotation angle is $\varphi=\arctan (\beta / \alpha)$. Using suitable interpolation functions, an interpolated array is generated where signal values are defined on new grid points. The whole scheme requires an input and an output interpolator. For an arbitrary angle, the values of $\alpha$ and $\beta$ may result inconveniently large, which might complicate the interpolation process.

The proposed design method gives another possible solution and is based on finding appropriate approximations for the two complex functions: $f_{1}\left(s_{1}\right)=e^{s_{1} \cos \varphi}, f_{2}\left(s_{2}\right)=e^{s_{2} \sin \varphi}$. These can be developed either in a power series (Taylor) or in a rational function using the Padé or Chebyshev-Padé approximations. We will first use the Padé approximation which has the advantage of yielding analytical expressions for the coefficients. We easily derive the following approximations, as for real variable functions:

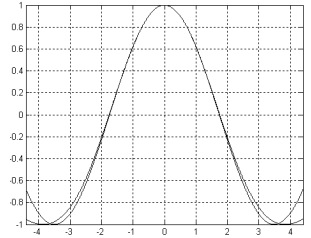

(a)

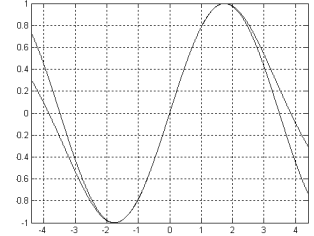

(b)

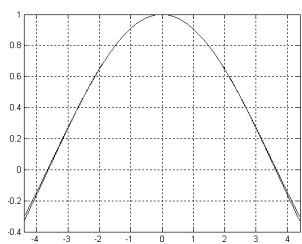

(c)

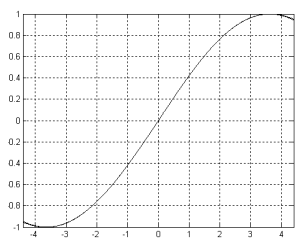

(d)

Fig. 1. Plots of exact functions vs. their approximations: (a) $\cos \left(\omega_{1} \cos \varphi\right) ;(b) \sin \left(\omega_{1} \cos \varphi\right)$;

(c) $\cos \left(\omega_{1} \sin \varphi\right) ;(d) \sin \left(\omega_{1} \sin \varphi\right)$

$$
\begin{aligned}
& \mathrm{f}_{1}\left(\mathrm{~s}_{1}\right) \cong\left(1+0.5 \cos \varphi \cdot \mathrm{s}_{1}+0.08333 \cos ^{2} \varphi \cdot \mathrm{s}_{1}^{2}\right) /\left(1-0.5 \cos \varphi \cdot \mathrm{s}_{1}+0.08333 \cos ^{2} \varphi \cdot \mathrm{s}_{1}^{2}\right)=\mathrm{f}_{\mathrm{a} 1}\left(\mathrm{~s}_{1}\right) \\
& \mathrm{f}_{2}\left(\mathrm{~s}_{2}\right) \cong\left(1+0.5 \sin \varphi \cdot \mathrm{s}_{2}+0.08333 \sin ^{2} \varphi \cdot \mathrm{s}_{2}^{2}\right) /\left(1-0.5 \sin \varphi \cdot \mathrm{s}_{2}+0.08333 \sin ^{2} \varphi \cdot \mathrm{s}_{2}^{2}\right)=\mathrm{f}_{\mathrm{a} 2}\left(\mathrm{~s}_{2}\right)
\end{aligned}
$$

Since $f_{1}\left(s_{1}\right)$ and $f_{2}\left(s_{2}\right)$ are complex functions $\left(s_{1}=j \omega_{1}, s_{2}=j \omega_{2}\right)$, the above approximations must hold separately for the real and imaginary parts, for instance:

$$
\operatorname{Re}\left[f_{1}\left(j \omega_{1}\right)\right]=\cos \left(\omega_{1} \cos \varphi\right) \cong \operatorname{Re}\left[f_{a 1}\left(j \omega_{1}\right)\right] \quad \operatorname{Im}\left[f_{1}\left(j \omega_{1}\right)\right]=\sin \left(\omega_{1} \cos \varphi\right) \cong \operatorname{Im}\left[f_{a 1}\left(j \omega_{1}\right)\right]
$$

In Fig. 1 we plotted comparatively the real and imaginary parts of the two complex functions $\mathrm{f}_{1}\left(\mathrm{~s}_{1}\right), \mathrm{f}_{2}\left(\mathrm{~s}_{2}\right)$ and of their rational approximations $\mathrm{f}_{\mathrm{a} 1}\left(\mathrm{~s}_{1}\right), \mathrm{f}_{\mathrm{a} 2}\left(\mathrm{~s}_{2}\right)$ given in (15). We notice that the proposed approximations are very accurate in the range $[-\pi, \pi]$. 
As shown in the following section, even using this low-order approximation a very good orientation-selective filter can be obtained. From the functions $f_{1}\left(s_{1}\right)$ and $f_{2}\left(s_{2}\right)$ we derive two corresponding discrete functions in the complex variables $z_{1}, z_{2}$. This can be achieved using the bilinear transform, a first-order approximation of the natural logarithm function. The sample interval can be taken $\mathrm{T}=1$ so the bilinear transform is $\mathrm{s}=2(\mathrm{z}-1) /(\mathrm{z}+1)$. Substituting it into relations (15), we obtain:

$$
\begin{array}{r}
F_{1}\left(z_{1}\right)=\frac{\left(1-\sin \varphi+0.4 \sin ^{2} \varphi\right) \cdot z_{1}^{-1}+\left(2-0.8 \sin ^{2} \varphi\right)+\left(1+\sin \varphi+0.4 \sin ^{2} \varphi\right) \cdot z_{1}}{\left(1+\sin \varphi+0.4 \sin ^{2} \varphi\right) \cdot z_{1}^{-1}+\left(2-0.8 \sin ^{2} \varphi\right)+\left(1-\sin \varphi+0.4 \sin ^{2} \varphi\right) \cdot z_{1}}=\frac{B_{1}\left(z_{1}\right)}{A_{1}\left(z_{1}\right)} \\
F_{2}\left(z_{2}\right)=\frac{\left(1-\cos \varphi+0.4 \cos ^{2} \varphi\right) \cdot z_{2}^{-1}+\left(2-0.8 \cos ^{2} \varphi\right)+\left(1+\cos \varphi+0.4 \cos ^{2} \varphi\right) \cdot z_{2}}{\left(1+\cos \varphi+0.4 \cos ^{2} \varphi\right) \cdot z_{2}^{-1}+\left(2-0.8 \cos ^{2} \varphi\right)+\left(1-\cos \varphi+0.4 \cos ^{2} \varphi\right) \cdot z_{2}}=\frac{B_{2}\left(z_{2}\right)}{A_{2}\left(z_{2}\right)}
\end{array}
$$

We used both negative and positive powers of $z_{1}$ and $z_{2}$ to put in evidence the coefficients symmetry. The function denoted $F_{\varphi}\left(z_{1}, z_{2}\right)$ will thus be the product of the above functions:

$$
\mathrm{F}_{\varphi}\left(\mathrm{z}_{1}, \mathrm{z}_{2}\right)=\mathrm{F}_{1}\left(\mathrm{z}_{1}\right) \cdot \mathrm{F}_{2}\left(\mathrm{z}_{2}\right)=\mathrm{B}_{\varphi}\left(\mathrm{z}_{1}, \mathrm{z}_{2}\right) / \mathrm{A}_{\varphi}\left(\mathrm{z}_{1}, \mathrm{z}_{2}\right)
$$

where $\mathrm{B}_{\varphi}\left(\mathrm{z}_{1}, \mathrm{z}_{2}\right)=\mathrm{B}_{1}\left(\mathrm{z}_{1}\right) \cdot \mathrm{B}_{2}\left(\mathrm{z}_{2}\right)$ and $\mathrm{A}_{\varphi}\left(\mathrm{z}_{1}, \mathrm{z}_{2}\right)=\mathrm{A}_{1}\left(\mathrm{z}_{1}\right) \cdot \mathrm{A}_{2}\left(\mathrm{z}_{2}\right)$.

An important remark here is that the derived frequency transformation is separable, as shows relation (19). Separability is a very desirable property of the 2D filter functions. However, the designed $2 \mathrm{D}$ oriented filters may not preserve this useful property.

Let $\mathbf{B}_{1}, \mathbf{B}_{2}, \mathbf{A}_{1}, \mathbf{A}_{2}$ be the coefficient vectors corresponding to $\mathrm{B}_{1}\left(\mathrm{z}_{1}\right), \mathrm{B}_{2}\left(\mathrm{z}_{2}\right), \mathrm{A}_{1}\left(\mathrm{z}_{1}\right)$, $A_{2}\left(z_{2}\right)$, identified from (17), (18) and $\mathbf{B}_{\varphi}, \mathbf{A}_{\varphi}$ the $3 \times 3$ matrices corresponding to $B_{\varphi}\left(z_{1}, z_{2}\right)$, $\mathbf{A}_{\varphi}\left(\mathrm{z}_{1}, \mathrm{z}_{2}\right)$. The matrices $\mathbf{B}_{\varphi}$ and $\mathbf{A}_{\varphi}$ of size $3 \times 3$ result as: $\mathbf{B}_{\varphi}=\mathbf{B}_{1}^{\mathrm{T}} \otimes \mathbf{B}_{2}, \mathbf{A}_{\varphi}=\mathbf{A}_{1}^{\mathrm{T}} \otimes \mathbf{A}_{2}$, where the upper index $\mathrm{T}$ denotes transposition and the symbol $\otimes$ outer product of vectors. The frequency transformation $\mathrm{z} \rightarrow \mathrm{F}_{\varphi}\left(\mathrm{z}_{1}, \mathrm{z}_{2}\right)$ can be finally expressed in the matrix form:

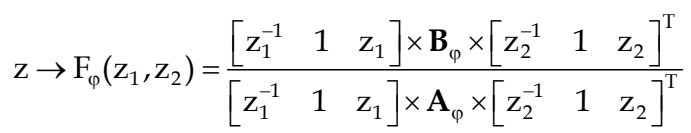

where $\times$ is matrix/vector product. Throughout the chapter we will use the term template, common in the field of cellular neural networks, referring to the coefficient matrices corresponding to the numerator and denominator of a $2 \mathrm{D}$ filter transfer function $\mathrm{H}\left(\mathrm{z}_{1}, \mathrm{z}_{2}\right)$. We will use mainly odd-sized templates (e.g. $3 \times 3,5 \times 5$ ) which correspond to even order filters and allow for using both positive and negative powers of $z_{1}$ and $z_{2}$.

Design example:

For an orientation angle $\varphi=\pi / 7$ we have $\sin \varphi=0.43389, \cos \varphi=0.90097$ and we obtain:

$$
z \rightarrow F_{\varphi}\left(z_{1}, z_{2}\right)=\frac{\left(0.6414 \cdot z_{1}^{-1}+1.8494+1.5092 \cdot z_{1}\right)}{\left(1.5092 \cdot z_{1}^{-1}+1.8494+0.6414 \cdot z_{1}\right)} \cdot \frac{\left(0.4237 \cdot z_{2}^{-1}+1.3506+2.2257 \cdot z_{2}\right)}{\left(2.2257 \cdot z_{2}^{-1}+1.3506+0.4237 \cdot z_{2}\right)}=\frac{B_{\varphi}\left(z_{1}, z_{2}\right)}{A_{\varphi}\left(z_{1}, z_{2}\right)}
$$


The numerator $\mathrm{B}_{\varphi}\left(\mathrm{z}_{1}, \mathrm{z}_{2}\right)$ and denominator $\mathrm{A}_{\varphi}\left(\mathrm{z}_{1}, \mathrm{z}_{2}\right)$ correspond to the $3 \times 3$ templates:

$$
\mathbf{B}_{\varphi}=\left[\begin{array}{lll}
0.271787 & 0.783643 & 0.639486 \\
0.866302 & 2.497802 & 2.038312 \\
1.427583 & 4.116139 & 3.358945
\end{array}\right] \quad \mathbf{A}_{\varphi}=\left[\begin{array}{lll}
3.358945 & 4.116139 & 1.427583 \\
2.038312 & 2.497802 & 0.866302 \\
0.639486 & 0.783643 & 0.271787
\end{array}\right]
$$

It is interesting to remark that matrix $\mathbf{B}_{\varphi}$ can be obtained from matrix $\mathbf{A}_{\varphi}$ by flipping successively the rows and columns of the matrix; so the matrix $\mathbf{B}_{\varphi}$ is the matrix $\mathbf{A}_{\varphi}$ rotated by $180^{\circ}$. The matrices have no symmetry, as the transfer function must result complex.

\subsection{Oriented Filter Design Using an 1D Prototype}

This section presents the design of an oriented filter based on an imposed 1D prototype. Let us consider a second-order digital filter with the transfer function in general form (3). Since we have found in the previous section the complex frequency transformation which leads to a 2D oriented filter from any $1 \mathrm{D}$ prototype transfer function in variable $\mathrm{z}$ :

$$
\mathrm{z} \rightarrow \mathrm{F}_{\varphi}\left(\mathrm{z}_{1}, \mathrm{z}_{2}\right)=\mathrm{B}_{\varphi}\left(\mathrm{z}_{1}, \mathrm{z}_{2}\right) / \mathrm{A}_{\varphi}\left(\mathrm{z}_{1}, \mathrm{z}_{2}\right)
$$

we only have to make the above substitution in $\mathrm{H}_{2}(\mathrm{z})$ given in (3) and we obtain the transfer function $\mathrm{H}_{\varphi}\left(\mathrm{z}_{1}, \mathrm{z}_{2}\right)$ of the desired oriented filter:

$$
H_{\varphi}\left(z_{1}, z_{2}\right)=\frac{b_{2} B_{\varphi}^{2}\left(z_{1}, z_{2}\right)+b_{1} A_{\varphi}\left(z_{1}, z_{2}\right) B_{\varphi}\left(z_{1}, z_{2}\right)+b_{0} A_{\varphi}^{2}\left(z_{1}, z_{2}\right)}{B_{\varphi}^{2}\left(z_{1}, z_{2}\right)+a_{1} A_{\varphi}\left(z_{1}, z_{2}\right) B_{\varphi}\left(z_{1}, z_{2}\right)+a_{0} A_{\varphi}^{2}\left(z_{1}, z_{2}\right)}
$$

For a chosen prototype of higher order, we get a similar rational function in powers of $\mathrm{A}_{\varphi}\left(\mathrm{z}_{1}, \mathrm{z}_{2}\right)$ and $\mathrm{B}_{\varphi}\left(\mathrm{z}_{1}, \mathrm{z}_{2}\right)$. Since the $2 \mathrm{D}$ transfer function (24) can be also described in terms of templates $\mathbf{B}, \mathbf{A}$ corresponding to its numerator and denominator, we have equivalently:

$$
\mathbf{B}=\mathrm{b}_{2} \cdot \mathbf{B}_{\varphi} * \mathbf{B}_{\varphi}+b_{1} \cdot \mathbf{A}_{\varphi} * \mathbf{B}_{\varphi}+b_{0} \cdot \mathbf{A}_{\varphi} * \mathbf{A}_{\varphi} \quad \mathbf{A}=\mathbf{B}_{\varphi} * \mathbf{B}_{\varphi}+a_{1} \cdot \mathbf{A}_{\varphi} * \mathbf{B}_{\varphi}+a_{0} \cdot \mathbf{A}_{\varphi} * \mathbf{A}_{\varphi}
$$

where * denotes two-dimensional convolution. The templates $\mathbf{A}$ and $\mathbf{B}$ result of size $5 \times 5$. The $2 \mathrm{D}$ oriented filter transfer function can be written generally in the matrix form:

$$
\mathrm{H}_{\varphi}\left(\mathrm{z}_{1}, \mathrm{z}_{2}\right)=\left(\mathbf{Z}_{1} \times \mathbf{B} \times \mathbf{Z}_{2}^{\mathrm{T}}\right) /\left(\mathbf{Z}_{1} \times \mathbf{A} \times \mathbf{Z}_{2}^{\mathrm{T}}\right)
$$

similar to expression (20), where:

$$
\mathbf{Z}_{1}=\left[\begin{array}{lllll}
\mathrm{z}_{1}^{-2} & \mathrm{z}_{1}^{-1} & 1 & \mathrm{z}_{1} & \mathrm{z}_{1}^{2}
\end{array}\right], \mathbf{Z}_{2}=\left[\begin{array}{lllll}
\mathrm{z}_{2}^{-2} & \mathrm{z}_{2}^{-1} & 1 & \mathrm{z}_{2} & \mathrm{z}_{2}^{2}
\end{array}\right]
$$

Generally, the 2D filter described by the templates B and A given in (25) is not strictly separable. However, the numerator and denominator of its transfer function are sums of 
separable terms. Since matrix convolution and outer product of vectors are commutative operations, using (25) we can express for instance the term:

$$
\mathbf{A}_{\varphi} * \mathbf{B}_{\varphi}=\left(\mathbf{A}_{1}^{\mathrm{T}} \otimes \mathbf{A}_{2}\right) *\left(\mathbf{B}_{1}^{\mathrm{T}} \otimes \mathbf{B}_{2}\right)=\left(\mathbf{A}_{1}^{\mathrm{T}} * \mathbf{B}_{1}^{\mathrm{T}}\right) \otimes\left(\mathbf{B}_{2} * \mathbf{B}_{2}\right)=\left(\mathbf{A}_{1} * \mathbf{B}_{1}\right)^{\mathrm{T}} \otimes\left(\mathbf{A}_{2} * \mathbf{B}_{2}\right)
$$

which is the outer product of two $1 \times 5$ vectors.

Design example. Next we design an oriented filter with specified parameters. We choose a very selective low-pass second-order digital filter. Let us consider an elliptic digital filter with parameters: pass-band ripple $R_{p}=0.1 \mathrm{~dB}$, stop-band attenuation $R_{s}=40 \mathrm{~dB}$ and very low passband-edge frequency $\omega_{\mathrm{p}}=0.02$ (1.0 is half the sampling frequency). The transfer function in $\mathrm{z}$ for this filter is:

$$
H_{p}(z)=\left(0.012277 \cdot z^{2}-0.012525 \cdot z+0.012277\right) /\left(z^{2}-1.850147 \cdot z+0.862316\right)
$$

The filter orientation angle is chosen $\varphi=\pi / 7$. Following the procedure described above the transfer function $\mathrm{H}_{\varphi}\left(\mathrm{z}_{1}, \mathrm{z}_{2}\right)$ results. Fig.2(a) shows the frequency response magnitude. As can be noticed, besides its central portion which looks correct, the filter also features some undesired portions located near the margins of the frequency plane. Also the characteristic tends to be distorted from the longitudinal axis near the frequency plane corners.

These errors are due to the approximation errors of the functions $f_{1}\left(s_{1}\right), f_{2}\left(s_{2}\right)$ near the ends of the frequency range and the distortions caused by the bilinear transform. In principle, if Padé approximations of higher order are used for $f_{1}\left(s_{1}\right)$ and $f_{2}\left(s_{2}\right)$, the errors will be reduced, but the price paid is an increased filter complexity.

The designed filter from Fig.2(a) cannot be used in this form, since it introduces large errors. However, a satisfactory oriented filter can be obtained by applying an additional wide-band low-pass filter which eliminates the distorted portions of the frequency characteristic. Such a "window" filter may be a maximally-flat circular filter, shown in Fig.2(b) and fully designed in (Matei \& Matei, 2009). Applying it we get the corrected directional filter whose frequency response and contour plot are given in Fig.2 (c) and (d).

A good oriented filter may be obtained as well using a Chebyshev-Padé approximation of the same order. For comparison, we will design again a filter with $\varphi=\pi / 7$. Using MAPLE we get the following approximation for $\mathrm{f}_{1}\left(\mathrm{~s}_{1}\right)=\exp \left(\mathrm{s}_{1} \cos (\pi / 7)\right)$ for $\omega \in[-\pi \sqrt{2}, \pi \sqrt{2}]$ :

$$
\mathrm{f}_{1}\left(\mathrm{~s}_{1}\right) \cong\left(1.355 \cdot \mathrm{T}\left(0, \mathrm{~s}_{0}\right)+1.823 \cdot \mathrm{T}\left(1, \mathrm{~s}_{0}\right)+0.56 \cdot \mathrm{T}\left(2, \mathrm{~s}_{0}\right)\right) /\left(\mathrm{T}\left(0, \mathrm{~s}_{0}\right)-1.184 \cdot \mathrm{T}\left(1, \mathrm{~s}_{0}\right)+0.256 \cdot \mathrm{T}\left(2, \mathrm{~s}_{0}\right)\right)(30)
$$

where $T\left(n, s_{0}\right)$ is a Chebyshev polynomial of order $n$ and $s_{0}=(1 / \pi \sqrt{2}) \cdot s=0.22727 \cdot s$. Substituting the expressions of the Chebyshev polynomials into (30), we get immediately:

$$
\mathrm{f}_{1}\left(\mathrm{~s}_{1}\right) \cong\left(1.0714+0.55723 \cdot \mathrm{s}_{1}+0.77598 \cdot \mathrm{s}_{1}^{2}\right) /\left(1-0.362 \cdot \mathrm{s}_{1}+0.035613 \cdot \mathrm{s}_{1}^{2}\right)
$$




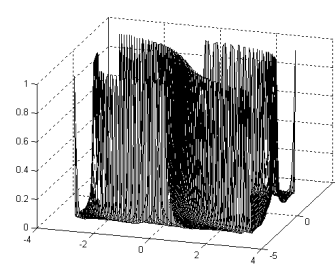

(a)

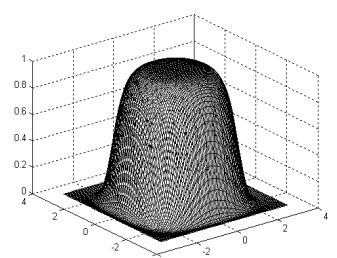

(b)

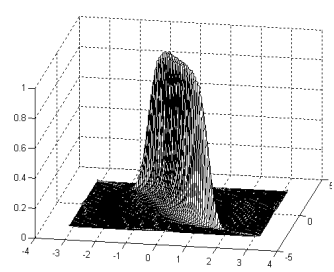

(c)

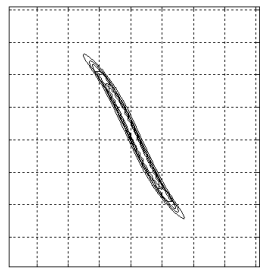

(d)

Fig. 2. (a) Uncorrected frequency response of the oriented filter; (b) circular window filter; (c) corrected filter frequency response; (d) contour plot

As before, in order to obtain a discrete approximation of $f_{1}\left(s_{1}\right)$, we use the bilinear transform and replace $s_{1}=2\left(z_{1}-1\right) /\left(z_{1}+1\right)$ in (31); we obtain the rational function:

$$
\mathrm{F}_{1}\left(\mathrm{z}_{1}\right)=\mathrm{B}_{1}\left(\mathrm{z}_{1}\right) / \mathrm{A}_{1}\left(\mathrm{z}_{1}\right)=\left(0.1559 \cdot \mathrm{z}_{1}^{-1}+0.8874+1.4555 \cdot \mathrm{z}_{1}\right) /\left(1.0885 \cdot \mathrm{z}_{1}^{-1}+1+0.244 \cdot \mathrm{z}_{1}\right)
$$

Similarly we get for $\mathrm{f}_{2}\left(\mathrm{~s}_{2}\right)=\exp \left(\mathrm{s}_{2} \sin (\pi / 7)\right)$ :

$$
\begin{gathered}
\mathrm{f}_{2}\left(\mathrm{~s}_{2}\right) \cong\left(1+0.224155 \cdot \mathrm{s}_{2}+0.015953 \cdot \mathrm{s}_{2}^{2}\right) /\left(1-0.208336 \cdot \mathrm{s}_{2}+0.013297 \cdot \mathrm{s}_{2}^{2}\right) \\
\mathrm{F}_{2}\left(\mathrm{z}_{2}\right)=\mathrm{B}_{2}\left(\mathrm{z}_{2}\right) / \mathrm{A}_{2}\left(\mathrm{z}_{2}\right)=\left(0.3259 \cdot \mathrm{z}_{2}^{-1}+0.9906+0.7994 \cdot \mathrm{z}_{2}\right) /\left(0.7762 \cdot \mathrm{z}_{2}^{-1}+1+0.3361 \cdot \mathrm{z}_{2}\right)
\end{gathered}
$$

We finally obtained the desired separable complex frequency transformation expressed as:

$$
\mathrm{z} \rightarrow \mathrm{F}_{\varphi}\left(\mathrm{z}_{1}, \mathrm{z}_{2}\right)=\mathrm{F}_{1}\left(\mathrm{z}_{1}\right) \cdot \mathrm{F}_{2}\left(\mathrm{z}_{2}\right)
$$

We denote $\mathbf{B}_{1}, \mathbf{B}_{2}, \mathbf{A}_{1}, \mathbf{A}_{2}$ the coefficient vectors corresponding to the numerators and denominators in (32) and (34). For instance we get from (32): $\mathbf{B}_{1}=\left[\begin{array}{llll}0.1559 & 0.8874 & 1.4555\end{array}\right]$. The matrices $\mathbf{B}_{\varphi}, \mathbf{A}_{\varphi}$ result as shown in section 3.1.

\section{Design example}

For comparison we have used the same prototype filter given by (29). The frequency response $\mathrm{H}_{\varphi}\left(\mathrm{z}_{1}, \mathrm{z}_{2}\right)$ results using (24); its magnitude from two views is shown in Fig.3(a), (b) and shows less parasitic portions as compared to the filter in Fig.2(a). Applying the same circular window filter, the characteristic is improved, as shown in Fig.3 (c),

The only drawback of the Chebyshev-Padé method is that, unlike Padé, cannot yield literal coefficient expressions in $\varphi$ as in (17), (18). Therefore, for each specified angle, the complex frequency transform $\mathrm{z} \rightarrow \mathrm{F}_{\varphi}\left(\mathrm{z}_{1}, \mathrm{z}_{2}\right)$ has to be calculated numerically.

The stability properties of this class of 2D IIR filters have still to be investigated. However, according to a theorem (Harn \& Shenoi, 1986), if $\mathrm{H}(Z)$ is a stable $1 \mathrm{D}$ recursive filter and $\mathrm{Z}=\mathrm{F}_{\varphi}\left(\mathrm{z}_{1}, \mathrm{z}_{2}\right)=\mathrm{F}_{1}\left(\mathrm{z}_{1}\right) \cdot \mathrm{F}_{2}\left(\mathrm{z}_{2}\right)$, where $\mathrm{F}_{1}\left(\mathrm{z}_{1}\right)$ and $\mathrm{F}_{2}\left(\mathrm{z}_{2}\right)$ are two stable DST (digital spectral transformation) functions, then $\mathrm{H}\left(\mathrm{F}_{1}\left(\mathrm{z}_{1}\right) \cdot \mathrm{F}_{2}\left(\mathrm{z}_{2}\right)\right)$ is also stable in the $\left(\mathrm{z}_{1}, \mathrm{z}_{2}\right)$ plane. The problem reduces to studying the stability of functions $F_{1}\left(z_{1}\right), F_{2}\left(z_{2}\right)$ of the form (17), (18). 
Here we approached the design of selective filters with a directional frequency response, but the method is more general and can be applied also to other types of prototype filters.

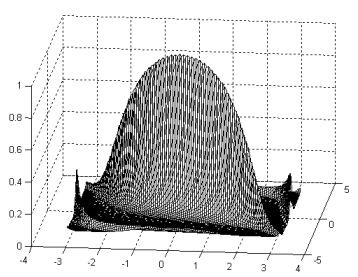

(a)

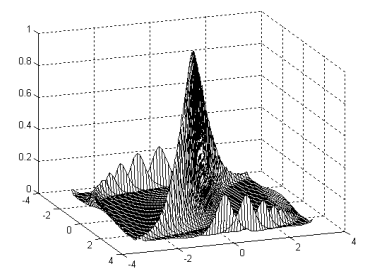

(b)

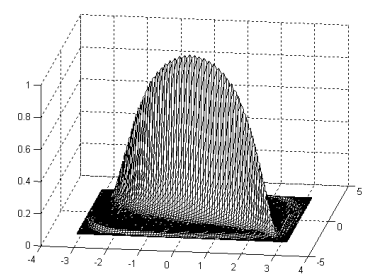

(c)

Fig. 3. (a), (b) Original oriented filter magnitude from two angles; (c) Oriented filter magnitude after applying the circular window filter

\section{Wedge-Shaped Filters}

Here we approach the design of a class of wedge filters in the 2D frequency domain, also treated in (Matei, 2009, a). We consider a general case of a wedge-shaped filter with a given orientation of its longitudinal axis. For design a maximally-flat 1D prototype filter will be used. We approach here only zero-phase filters, often preferred in image filtering due to the absence of phase distortions. Two ideal wedge filters in the frequency plane are shown in Fig.4. The filter in Fig.4 (a) has its frequency response along the axis $\omega_{2}$. The angle $\Varangle \mathrm{AOB}=\theta$ will be referred to as aperture angle. In Fig.4 (b) a more general wedge filter is shown, with aperture angle $\Varangle \mathrm{BOD}=\theta$, oriented along an axis $\mathrm{CC}^{\prime}$, forming an angle $\Varangle \mathrm{AOC}=\varphi$ with frequency axis $\mathrm{O}-\omega_{2}$.

The Bamberger directional filter bank (Bamberger \& Smith, 1992) is an angularly oriented image decomposition that splits the 2D frequency plane into wedge-shape channels with $\mathrm{N}$ $=2,4,6$, and 8 sub-bands (channels). Each sub-band captures spatial detail along a specific orientation. In Fig. 5 the frequency band partitions are shown for $\mathrm{N}=8$.

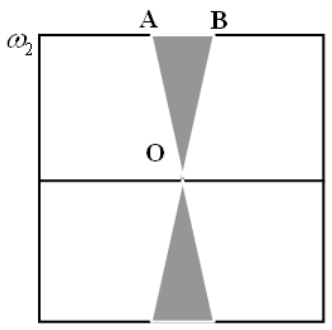

(a)

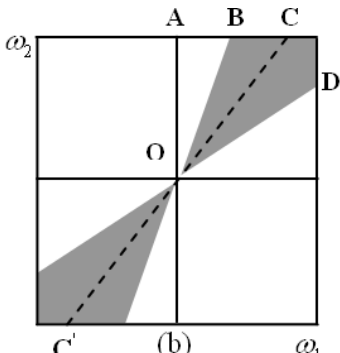

Fig. 4. Ideal wedge filters: (a) along the axis $\omega_{2} ;(b)$ oriented at an angle $\varphi$

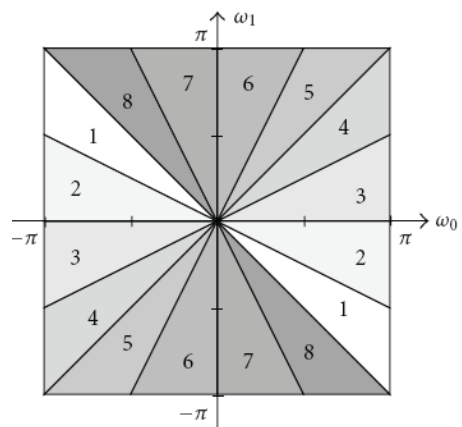

Fig. 5. 8-band partitions of the frequency plane 


\subsection{Wedge Filter Design Using Frequency Transformations}

Next we present a design method which leads to 2D zero-phase oriented filters from $1 D$ prototypes. Let us consider a 1D IIR zero-phase low-pass filter frequency response:

$$
\mathrm{H}_{\mathrm{p}}(\omega)=\left(\mathrm{b}_{0}+\mathrm{b}_{1} \omega^{2}+\mathrm{b}_{2} \omega^{4}\right) /\left(1+\mathrm{a}_{1} \omega^{2}+\mathrm{a}_{2} \omega^{4}\right)
$$

where usually $b_{0}=H_{p}(0)=1$, obtained as in section 2, with general expression (8). A wedge filter along frequency axis $\omega_{2}$ can be obtained using the $1 \mathrm{D}$ to $2 \mathrm{D}$ frequency transformation:

$$
\omega \rightarrow \mathrm{f}\left(\omega_{1}, \omega_{2}\right)=\mathrm{a} \cdot \omega_{1} / \omega_{2} \quad\left(\text { for } \omega_{2} \neq 0\right)
$$

We denoted $\mathrm{a}=1 / \operatorname{tg}(\theta / 2)$, where $\theta$ is the aperture angle of the wedge filter, as defined in Fig.4. Replacing in (36) $\omega$ by the ratio a $\omega_{1} / \omega_{2}$, we get the frequency response in $\omega_{1}, \omega_{2}$ :

$$
\mathrm{H}\left(\omega_{1}, \omega_{2}\right)=\left(b_{0} \omega_{2}^{4}+b_{1} a^{2} \omega_{1}^{2} \omega_{2}^{2}+b_{2} a^{4} \omega_{1}^{4}\right) /\left(\omega_{2}^{4}+a_{1} a^{2} \omega_{1}^{2} \omega_{2}^{2}+a_{2} a^{4} \omega_{1}^{4}\right)
$$

At this point we map $H\left(\omega_{1}, \omega_{2}\right)$ into the complex plane $\left(s_{1}, s_{2}\right)$, where $s_{1}=j \omega_{1}, s_{2}=j \omega_{2}$. Since $\omega_{1}^{2}=-\mathrm{s}_{1}^{2}$ and $\omega_{2}^{2}=-\mathrm{s}_{2}^{2}$ we get the function $\mathrm{H}_{\mathrm{S}}\left(\mathrm{s}_{1}, \mathrm{~s}_{2}\right)$ :

$$
\mathrm{H}_{\mathrm{S}}\left(\mathrm{s}_{1}, \mathrm{~s}_{2}\right)=\left(\mathrm{b}_{0} \mathrm{~s}_{2}^{4}+\mathrm{b}_{1} \mathrm{a}^{2} \mathrm{~s}_{1}^{2} \mathrm{~s}_{2}^{2}+\mathrm{b}_{2} \mathrm{a}^{4} \mathrm{~s}_{1}^{4}\right) /\left(\mathrm{s}_{2}^{4}+\mathrm{a}_{1} \mathrm{a}^{2} \mathrm{~s}_{1}^{2} \mathrm{~s}_{2}^{2}+\mathrm{a}_{2} \mathrm{a}^{4} \mathrm{~s}_{1}^{4}\right)
$$

A little more difficult task is now to find a mapping of $\mathrm{H}_{\mathrm{S}}\left(\mathrm{s}_{1}, \mathrm{~s}_{2}\right)$ into the complex plane $\left(z_{1}, z_{2}\right)$. This can be achieved either using the forward or backward Euler approximations, or otherwise the bilinear transform, which gives better accuracy. The bilinear transform for $\mathrm{s}_{1}$ and $\mathrm{s}_{2}$ in the complex plane $\left(\mathrm{s}_{1}, \mathrm{~s}_{2}\right)$ has the form:

$$
\mathrm{s}_{1}=2\left(\mathrm{z}_{1}-1\right) /\left(\mathrm{z}_{1}+1\right) \quad \mathrm{s}_{2}=2\left(\mathrm{z}_{2}-1\right) /\left(\mathrm{z}_{2}+1\right)
$$

Substituting $s_{1}, s_{2}$ in (39), we find after some algebra a function in $z_{1}, z_{2}$ in matrix form:

$$
\mathrm{F}\left(\mathrm{z}_{1}, \mathrm{z}_{2}\right)=\left(\mathbf{Z}_{1} \times \mathbf{B} \times \mathbf{Z}_{2}^{\mathrm{T}}\right) /\left(\mathbf{Z}_{1} \times \mathbf{A} \times \mathbf{Z}_{2}^{\mathrm{T}}\right)
$$

where $\mathbf{Z}_{1}$ and $\mathbf{Z}_{2}$ are the vectors given by (27) and $\times$ denotes matrix/vector product. The filter templates $\mathbf{B}$ and $\mathbf{A}$ can be written as a sum of three separable matrices:

$$
\begin{aligned}
& \mathbf{B}=\mathrm{b}_{0} \cdot \mathbf{M}_{1}^{\mathrm{T}} * \mathbf{M}_{2}+\mathrm{b}_{1} \mathrm{a}^{2} \cdot \mathbf{M}_{3}^{\mathrm{T}} * \mathbf{M}_{3}+\mathrm{b}_{2} \mathrm{a}^{4} \cdot \mathbf{M}_{2}^{\mathrm{T}} * \mathbf{M}_{1} \\
& \mathbf{A}=\mathbf{M}_{1}^{\mathrm{T}} * \mathbf{M}_{2}+\mathrm{a}_{1} \mathrm{a}^{2} \cdot \mathbf{M}_{3}^{\mathrm{T}} * \mathbf{M}_{3}+\mathrm{a}_{2} \mathrm{a}^{4} \cdot \mathbf{M}_{2}^{\mathrm{T}} * \mathbf{M}_{\mathbf{1}}
\end{aligned}
$$

where $\mathbf{M}_{1}, \mathbf{M}_{2}$ and $\mathbf{M}_{3}$ are row vectors: $\mathbf{M}_{1}=\left[\begin{array}{lllll}1 & 4 & 6 & 4 & 1\end{array}\right], \mathbf{M}_{2}=\left[\begin{array}{lllll}1 & -4 & 6 & -4 & 1\end{array}\right]$, $\mathbf{M}_{3}=\left[\begin{array}{lllll}1 & 0 & -2 & 0 & 1\end{array}\right]$ and the operator $*$ denotes outer product of vectors. 
In a more general case when the wedge filter axis has an orientation specified by an angle $\varphi$ (with respect to the axis $\omega_{2}$ ), the oriented wedge filter may be obtained by rotating the axes of the plane $\left(\omega_{1}, \omega_{2}\right)$ with an angle $\varphi$. The rotation is defined by the linear transformation (12). In this case the $1 \mathrm{D}$ to $2 \mathrm{D}$ frequency transformation can be written as:

$$
\omega \rightarrow \mathrm{f}_{\varphi}\left(\omega_{1}, \omega_{2}\right)=\mathrm{a}\left(\omega_{1}-\omega_{2} \cdot \operatorname{tg} \varphi\right) /\left(\omega_{1} \cdot \operatorname{tg} \varphi+\omega_{2}\right)
$$

Using the expression above and the bilinear transform, we finally get a mapping of the form:

$$
\omega^{2} \rightarrow \mathrm{F}\left(\mathrm{z}_{1}, \mathrm{z}_{2}\right)=\mathrm{a}^{2} \cdot\left(\mathbf{z}_{1} \times \mathbf{M}_{\varphi} \times \mathbf{z}_{2}^{\mathrm{T}}\right) /\left(\mathbf{z}_{1} \times \mathbf{M}_{\varphi}^{90^{0}} \times \mathbf{z}_{2}^{\mathrm{T}}\right)
$$

where $\mathbf{z}_{1}=\left[\begin{array}{lll}z_{1}^{-1} & 1 & z_{1}\end{array}\right], \mathbf{z}_{2}=\left[\begin{array}{lll}z_{2}^{-1} & 1 & z_{2}\end{array}\right]$ and $\mathbf{M}_{\varphi}$ is the $3 \times 3$ matrix:

$$
\mathbf{M}_{\varphi}=\left[\begin{array}{ccc}
(\operatorname{tg} \varphi-1)^{2} & 2\left(\operatorname{tg}^{2} \varphi-1\right) & (\operatorname{tg} \varphi+1)^{2} \\
-2\left(\operatorname{tg}^{2} \varphi-1\right) & -4\left(\operatorname{tg}^{2} \varphi+1\right) & -2\left(\operatorname{tg}^{2} \varphi-1\right) \\
(\operatorname{tg} \varphi+1)^{2} & 2\left(\operatorname{tg}^{2} \varphi-1\right) & (\operatorname{tg} \varphi-1)^{2}
\end{array}\right]
$$

and $\mathbf{M}_{\varphi}^{90^{0}}$ is the matrix $\mathbf{M}_{\varphi}$ rotated by $90^{\circ}$. Applying this frequency transformation directly to the $1 D$ prototype (36), we get the $2 \mathrm{D}$ wedge filter transfer function in $z_{1}, z_{2}$ :

$$
\mathrm{H}_{\varphi}\left(\mathrm{z}_{1}, \mathrm{z}_{2}\right)=\left(\mathbf{Z}_{1} \times \mathbf{B}_{\varphi} \times \mathbf{Z}_{2}^{\mathrm{T}}\right) /\left(\mathbf{Z}_{1} \times \mathbf{A}_{\varphi} \times \mathbf{Z}_{2}^{\mathrm{T}}\right)
$$

where the $5 \times 5$ matrices $\mathbf{A}_{\varphi}$ and $\mathbf{B}_{\varphi}$ have the expressions:

$$
\begin{gathered}
\mathbf{B}_{\varphi}=\mathrm{b}_{0}\left(\mathbf{M}_{\varphi} * \mathbf{M}_{\varphi}\right)^{90^{0}}+\mathrm{b}_{1} \mathrm{a}^{2}\left(\mathbf{M}_{\varphi} * \mathbf{M}_{\varphi}^{90^{0}}\right)+\mathrm{b}_{2} \mathrm{a}^{4}\left(\mathbf{M}_{\varphi} * \mathbf{M}_{\varphi}\right) \\
\mathbf{A}_{\varphi}=\left(\mathbf{M}_{\varphi} * \mathbf{M}_{\varphi}\right)^{90^{0}}+\mathrm{a}_{1} \mathrm{a}^{2}\left(\mathbf{M}_{\varphi} * \mathbf{M}_{\varphi}^{90^{0}}\right)+\mathrm{a}_{2} \mathrm{a}^{4}\left(\mathbf{M}_{\varphi} * \mathbf{M}_{\varphi}\right)
\end{gathered}
$$

and $Z_{1}$ and $Z_{2}$ are the vectors given in (27). Therefore the transfer function $H_{\varphi}\left(z_{1}, z_{2}\right)$ in (46) corresponds to a wedge filter with an aperture angle $\theta=2 \cdot \operatorname{arctg}(1 / a)$ and whose longitudinal axis is tilted about the $\omega_{2}$ axis in the frequency plane with an angle $\varphi$.

Even if this method is straightforward and easy to apply once found the 1D prototype filter, the designed 2D filter will present noticeable distortions towards the limits of the frequency plane as compared to the ideal frequency response (38). This is mainly due to the frequency warping effect introduced by the bilinear transform, expressed by the continuous-time to discrete-time frequency mapping:

$$
\omega=(2 / \mathrm{T}) \cdot \operatorname{arctg}\left(\omega_{\mathrm{a}} \mathrm{T} / 2\right)
$$


where $\omega$ is the frequency of the discrete-time filter and $\omega_{a}$ the frequency of the continuoustime filter. In order to correct this distortion we next apply a pre-warping, using the inverse of mapping (49). For our purposes we can take $T=1$ and we substitute $\omega_{1} \rightarrow 2 \operatorname{tg}\left(\omega_{1} / 2\right)$, $\omega_{2} \rightarrow 2 \operatorname{tg}\left(\omega_{2} / 2\right)$ in (43). Since these are nonlinear mappings, a polynomial or rational approximation would be more suitable. Using a Chebyshev-Padé approximation we get:

$$
\operatorname{tg}(\omega / 2) \cong \omega \cdot\left(0.5-0.008439 \cdot \omega^{2}\right) /\left(1-0.1 \cdot \omega^{2}\right)=g(\omega)
$$

very accurate on a frequency range close to $[-\pi, \pi]$. Using (43) we obtain the frequency transformation which includes frequency pre-warping for $\omega_{1}$ and $\omega_{2}$ :

$$
\omega \rightarrow \mathrm{f}_{\varphi \mathrm{P}}\left(\omega_{1}, \omega_{2}\right)=\mathrm{a}\left(\operatorname{tg}\left(\omega_{1} / 2\right)-\operatorname{tg}\left(\omega_{2} / 2\right) \cdot \operatorname{tg} \varphi\right) /\left(\operatorname{tg}\left(\omega_{1} / 2\right) \cdot \operatorname{tg} \varphi+\operatorname{tg}\left(\omega_{2} / 2\right)\right)
$$

Substituting in (51) $\operatorname{tg}(\omega / 2)$ by the rational approximation $\mathrm{g}(\omega)$ we get a rational expression in $\omega_{1}$ and $\omega_{2}$ for the frequency transformation $\omega \rightarrow f_{\varphi P}\left(\omega_{1}, \omega_{2}\right)$. Then as previously we map $\mathrm{f}_{\varphi \mathrm{P}}\left(\omega_{1}, \omega_{2}\right)$ into the complex plane $\left(\mathrm{s}_{1}, \mathrm{~s}_{2}\right)$ and finally we get using bilinear transform the frequency mapping written again in matrix form: $F: \mathbb{R} \rightarrow \mathbb{C}^{2}, \omega \rightarrow F\left(z_{1}, z_{2}\right)$

$$
\mathrm{F}\left(\mathrm{z}_{1}, \mathrm{z}_{2}\right)=\left(\mathbf{Z}_{1} \times \mathbf{B}_{\mathrm{P \varphi}} \times \mathbf{Z}_{2}^{\mathrm{T}}\right) /\left(\mathbf{Z}_{1} \times \mathbf{A}_{\mathrm{P} \varphi} \times \mathbf{Z}_{2}^{\mathrm{T}}\right)
$$

The $4 \times 4$ templates corresponding to the numerator and denominator have the form:

$$
\mathbf{B}_{\mathrm{p} \varphi}=\mathbf{M}_{1}-\operatorname{tg} \varphi \cdot \mathbf{M}_{1}^{90^{0}} \quad \mathbf{A}_{\mathrm{p} \varphi}=\operatorname{tg} \varphi \cdot \mathbf{M}_{1}+\mathbf{M}_{1}^{90^{0}}
$$

where $\mathbf{M}_{1}^{90^{0}}$ is the matrix $\mathbf{M}_{1}$ rotated clock-wise by $90^{\circ}$, numerically given by:

$$
\mathbf{M}_{1}=\left[\begin{array}{ll}
-1 & 1 \\
-1 & 1
\end{array}\right] *\left[\begin{array}{lll}
0.559283 & 1.081434 & 0.559283 \\
0.915190 & 1.769619 & 0.915190 \\
0.559283 & 1.081434 & 0.559283
\end{array}\right]
$$

The elements of $\mathbf{M}_{1}$ result from combinations of the coefficients occurring in the expression of $g(\omega)$ in (50). Finally we obtain the 1D to 2D frequency transformation in the matrix form:

$$
\omega^{2} \rightarrow \mathrm{F}\left(\mathrm{z}_{1}, \mathrm{z}_{2}\right)=\mathrm{a}^{2} \cdot\left(\mathbf{z}_{1} \times \mathbf{B} \times \mathbf{z}_{2}^{\mathrm{T}}\right) /\left(\mathbf{z}_{1} \times \mathbf{A} \times \mathbf{z}_{2}^{\mathrm{T}}\right)
$$

where the matrices $\mathbf{B}=\mathbf{B}_{\mathrm{p} \varphi} * \mathbf{B}_{\mathrm{p} \varphi}, \mathbf{A}=\mathbf{A}_{\mathrm{p} \varphi} * \mathbf{A}_{\mathrm{p} \varphi}$ resulted by convolution are of size $7 \times 7$. We can apply this frequency transformation directly to the 1D prototype function (36) and we obtain the $2 D$ wedge filter transfer function in $z_{1}$ and $z_{2}$ : 


$$
\mathrm{H}_{\mathrm{W} \varphi}\left(\mathbf{z}_{1}, \mathbf{z}_{2}\right)=\left(\mathbf{Z}_{1} \times \mathbf{B}_{\mathbf{W}_{\varphi}} \times \mathbf{Z}_{2}^{\mathrm{T}}\right) /\left(\mathbf{Z}_{1} \times \mathbf{A}_{W_{\varphi}} \times \mathbf{Z}_{2}^{\mathrm{T}}\right)
$$

where $\mathbf{Z}_{1}$ and $\mathbf{Z}_{2}$ are row vectors: $\mathbf{Z}_{1}=\left[\begin{array}{llllllll}\mathrm{z}_{1}^{\mathrm{N}} & \mathrm{z}_{1}^{\mathrm{N}-1} & \ldots & \mathrm{z}_{1} & 1\end{array}\right], \quad \mathbf{Z}_{2}=\left[\begin{array}{llllll}\mathrm{z}_{2}^{\mathrm{N}} & \mathrm{z}_{2}^{\mathrm{N}-1} & \ldots & \mathrm{z}_{2} & 1\end{array}\right]$ with $\mathrm{N}=12$; the $13 \times 13$ matrices $\mathbf{A}_{\mathbf{W}_{\varphi}}$ and $\mathbf{B}_{\mathbf{W}_{\varphi}}$ are:

$$
\mathbf{B}_{\mathrm{W}_{\varphi}}=\mathrm{b}_{0}(\mathbf{A} * \mathbf{A})+\mathrm{b}_{1} \mathrm{a}^{2}(\mathbf{A} * \mathbf{B})+\mathrm{b}_{2} \mathrm{a}^{4}(\mathbf{B} * \mathbf{B}), \quad \mathbf{A}_{\mathbf{W} \varphi}=\mathbf{A} * \mathbf{A}+\mathrm{a}_{1} \mathrm{a}^{2}(\mathbf{A} * \mathbf{B})+\mathrm{a}_{2} \mathrm{a}^{4}(\mathbf{B} * \mathbf{B})
$$

As an important remark, even if the filter templates result relatively large, this is the price paid for ensuring a good linearity of the filter shape in the frequency plane. The frequency pre-warping has increased the filter order. However, the filter large-size templates result as a convolution of small size matrices $(3 \times 3,5 \times 5)$ and can be considered partially separable. At least the numerator of the prototype (36) may have real roots, so it can be factorized, which implies convolution of smaller size matrices. Let us consider the maximally-flat zerophase 1D IIR prototype filter shown in Fig.6 (a), with the transfer function:

$$
\mathrm{H}_{\mathrm{p}}(\mathrm{s})=\left(0.887175+0.269975 \cdot \mathrm{s}^{2}+0.018905 \cdot \mathrm{s}^{4}\right) /\left(1+0.600346 \cdot \mathrm{s}^{2}+5.332057 \cdot \mathrm{s}^{4}\right)
$$

Using this prototype, we designed a wedge filter with an aperture angle $\theta=0.2 \pi$ and orientation angle $\varphi=\pi / 5$. For these values we get $a=\operatorname{tg}(\theta / 2)=0.3249, \operatorname{tg} \varphi=0.7265$. The frequency response and contour plot are shown in Fig.6 (b) and (c).

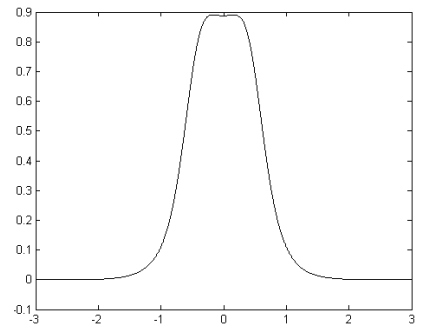

(a)

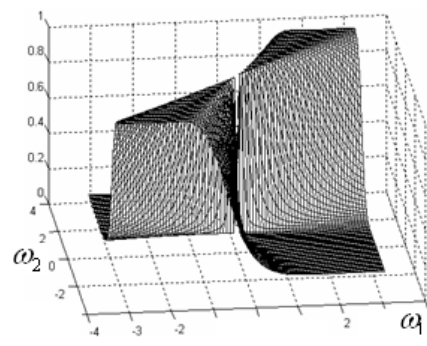

(b)

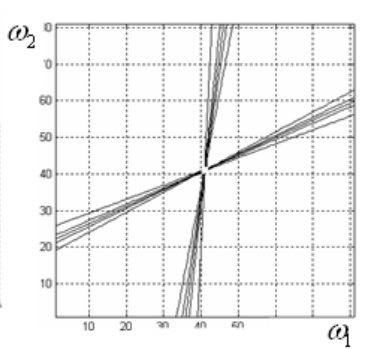

(c)

Fig. 6. Oriented flat-top wedge filter with $\theta=0.2 \pi$ and $\varphi=0.2 \pi$ : (a) $1 \mathrm{D}$ IIR maximally-flat prototype magnitude; (b) frequency response; (c) contour plot

\subsection{Design Method Using Numerical Approximation}

The second design method for zero-phase wedge-shaped filters starts again from a zerophase $1 \mathrm{D}$ prototype filter of the general form similar to (36). We will use again the $1 \mathrm{D}$ to $2 \mathrm{D}$ frequency mapping (43). Since (36) is a rational function of $\omega^{2}$, the design method will be based upon finding the discrete approximation of the function

$$
\mathrm{F}_{\varphi}\left(\omega_{1}, \omega_{2}\right)=\mathrm{f}_{\varphi}^{2}\left(\omega_{1}, \omega_{2}\right)=\mathrm{a}^{2}\left(\omega_{1}-\omega_{2} \cdot \operatorname{tg} \varphi\right)^{2} /\left(\omega_{1} \cdot \operatorname{tg} \varphi+\omega_{2}\right)^{2}
$$


This approximation will be derived indirectly, using the change of variables: $\omega_{1}=\arccos x_{1}$, $\omega_{2}=\arccos x_{2}$ and the function $F_{\varphi}\left(\omega_{1}, \omega_{2}\right)$ will be mapped into a function $G_{\varphi}\left(x_{1}, x_{2}\right)$. The next step is to find a two-variable Taylor series expansion of the function $G_{\varphi}\left(x_{1}, x_{2}\right)$. Using a symbolic calculation software like MAPLE, we easily determine this series expansion in the variables $x_{1}, x_{2}$. Then we return to the former variables by substituting back $x_{1}=\cos \omega_{1}$, $x_{2}=\cos \omega_{2}$ in $G_{\varphi}\left(x_{1}, x_{2}\right)$. Thus we obtain an approximation of $F_{\varphi}\left(\omega_{1}, \omega_{2}\right)$ in powers of $\cos \omega_{1}, \cos \omega_{2}$. Using trigonometric identities, we finally express $F_{\varphi}\left(\omega_{1}, \omega_{2}\right)$ as:

$$
\mathrm{F}_{\varphi}\left(\omega_{1}, \omega_{2}\right) \cong \sum_{\mathrm{m}=-\mathrm{N}}^{\mathrm{N}} \sum_{\mathrm{n}=-\mathrm{N}}^{\mathrm{N}} \mathrm{a}_{\mathrm{mn}} \cdot \cos \left(\mathrm{m} \omega_{1}+\mathrm{n} \omega_{2}\right)
$$

where $N$ is chosen to ensure a desired precision (usually $\mathrm{N}=2$ ). The coefficients $\mathrm{a}_{\mathrm{mn}}$ depend on the orientation angle $\varphi$ and they are polynomial expressions in the variable $\operatorname{tg} \varphi$. Let us design a wedge filter with the same specifications from section 4.1, i.e. the prototype (58), with the parameters: $\mathrm{a}=\operatorname{tg}(\theta / 2)=0.3249, \operatorname{tg} \varphi=0.7265$. The proposed method yields:

$$
\begin{aligned}
& \mathrm{F}_{\varphi}\left(\omega_{1}, \omega_{2}\right) \cong \mathrm{a}^{2} \cdot\left[0.195736-0.132213 \cdot \cos \left(\omega_{1}\right)+0.212134 \cdot \cos \left(\omega_{2}\right)-0.155057 \cdot\left(\cos \left(\omega_{1}-\omega_{2}\right)\right.\right. \\
& \left.+\cos \left(\omega_{1}+\omega_{2}\right)\right)-0.027075 \cdot\left(\cos \left(2 \omega_{1}-\omega_{2}\right)+\cos \left(2 \omega_{1}+\omega_{2}\right)\right)-0.042024 \cdot\left(\cos \left(\omega_{1}-2 \omega_{2}\right)\right. \\
& \left.\left.+\cos \left(\omega_{1}+2 \omega_{2}\right)\right)+0.050075 \cos \left(2 \omega_{1}\right)+0.124584 \cos \left(2 \omega_{2}\right)-0.014742 \cdot\left(\cos \left(2 \omega_{1}-2 \omega_{2}\right)+\cos \left(2 \omega_{1}+2 \omega_{2}\right)\right)\right]
\end{aligned}
$$

which corresponds to the $5 \times 5$ template:

$$
\mathbf{W}=\mathrm{a}^{2} \cdot\left[\begin{array}{rrrrr}
-0.0073 & -0.0210 & 0.0623 & -0.0210 & -0.0073 \\
-0.0135 & -0.0775 & 0.1060 & -0.0775 & -0.0135 \\
0.0250 & -0.0661 & 0.1957 & -0.0661 & 0.0250 \\
-0.0135 & -0.0775 & 0.1060 & -0.0775 & -0.0135 \\
-0.0073 & -0.0210 & 0.0623 & -0.0210 & -0.0073
\end{array}\right]
$$

found after identifying coefficients of the $2 \mathrm{D} Z$ transform corresponding to (61). Once obtained the $1 \mathrm{D}$ to $2 \mathrm{D}$ frequency mapping of the form: $\omega^{2} \rightarrow \mathrm{F}_{\varphi}\left(\omega_{1}, \omega_{2}\right)$ given by the expression (61), the next design step is straightforward and consists simply in substituting in $\mathrm{H}_{\mathrm{p}}(\omega)$ from (36) $\omega^{2}$ with $\mathrm{F}_{\varphi}\left(\omega_{1}, \omega_{2}\right)$. The templates $\mathbf{B}$ and $\mathbf{A}$ of the wedge filter result according to the numerator and denominator of $\mathrm{H}_{\mathrm{p}}(\omega)$ as:

$$
\mathbf{B}=\mathrm{b}_{0} \cdot \mathbf{E}+\mathrm{b}_{1} \cdot \mathbf{W}_{\mathbf{b}}+\mathrm{b}_{2} \cdot \mathbf{W} * \mathbf{W} \quad \mathbf{A}=\mathbf{E}+\mathrm{a}_{1} \cdot \mathbf{W}_{\mathbf{b}}+\mathrm{a}_{2} \cdot \mathbf{W} * \mathbf{W}
$$

where * stands for matrix convolution and $\mathrm{E}$ is a $9 \times 9$ matrix with zero elements and the central element 1 . The $9 \times 9$ matrix $\mathbf{W}_{\mathbf{b}}$ is obtained by bordering the $5 \times 5$ matrix $\mathbf{W}$ with zeros in order to be summed with matrices $\mathbf{E}$ and $\mathbf{W} * \mathbf{W}$. 
An advantage of the second design method is that it avoids the use of the bilinear transform, which is known to introduce distortions unless a frequency pre-warping is applied, as in the previous section. The pre-warping increases the filter complexity, as shown. As a general remark, the second design approach is somewhat simpler than the first but requires the use of bivariate Taylor series expansion for a given orientation angle $\varphi$.

\subsection{Fan Filters Design}

Although there exist design methods for FIR or IIR fan filters (Kayran \& King, 1983), they can be derived as well using the proposed method. We consider two types of fan filters specified in the plane $\left(\omega_{1}, \omega_{2}\right)$ as in Fig.7 (a), (b). The filter in Fig.7 (a) can be described ideally as:

$$
\mathrm{H}_{\mathrm{F}}\left(\omega_{1}, \omega_{2}\right)= \begin{cases}1, & \left|\omega_{2}\right| \leq\left|\omega_{1}\right| \\ 0, & \text { otherwise }\end{cases}
$$

This fan filter is a particular case of a wedge filter with the aperture angle $\theta=\pi / 2$ and orientation $\varphi=0$, therefore $\mathrm{a}=1$ and $\operatorname{tg} \varphi=0$; the frequency transformation (43) reduces to the simple expression $\omega \rightarrow \mathrm{f}_{\varphi}\left(\omega_{1}, \omega_{2}\right)=\omega_{1} / \omega_{2}$. In this particular case the template $\mathbf{W}$ results:

$$
\mathbf{W}=\left[\begin{array}{lllll}
0.0072 & -0.0413 & 0.1038 & -0.0413 & 0.0072 \\
0.0134 & -0.1056 & 0.1746 & -0.1056 & 0.0134 \\
0.0281 & -0.1474 & 0.2975 & -0.1474 & 0.0281 \\
0.0134 & -0.1056 & 0.1746 & -0.1056 & 0.0134 \\
0.0072 & -0.0413 & 0.1038 & -0.0413 & 0.0072
\end{array}\right]
$$

The frequency response of a fan filter of this type, using the above specifications and the prototype given in (58), is shown in Fig.7 (c). We notice that it preserves the 1D prototype maximally-flat characteristics in the pass-band.

For the second fan filter type in Fig.7 (b) we have the parameters: $\theta=\pi / 2$ and $\varphi=\pi / 4$, therefore $\mathrm{a}=1$ and $\operatorname{tg} \theta=1$; in this case the frequency transformation (43) simplifies to:

$$
\omega \rightarrow \mathrm{f}_{\varphi}\left(\omega_{1}, \omega_{2}\right)=\left(\omega_{1}-\omega_{2}\right) /\left(\omega_{1}+\omega_{2}\right)
$$

In this particular case the template $\mathbf{W}$ results as:

$$
\mathbf{W}=\left[\begin{array}{rrrrr}
-0.0071 & -0.0126 & 0.0383 & -0.0126 & -0.0071 \\
-0.0126 & -0.0681 & 0.0131 & -0.0681 & -0.0126 \\
0.0383 & 0.0131 & 0.0760 & 0.0131 & 0.0383 \\
-0.0126 & -0.0681 & 0.0131 & -0.0681 & -0.0126 \\
-0.0071 & -0.0126 & 0.0383 & -0.0126 & -0.0071
\end{array}\right]
$$

The filter templates result again using relations (63). 


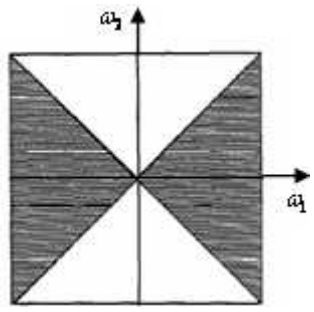

(a)

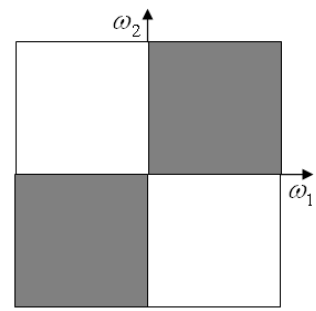

(b)

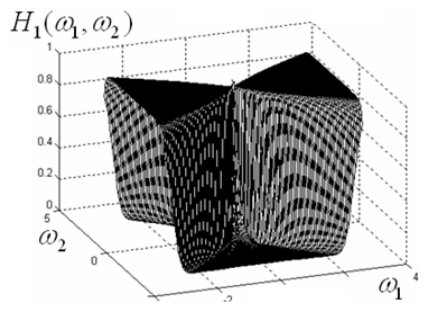

(c)

Fig. 7. (a), (b) Two versions of ideal fan filters; (c) fan filter frequency response

\section{2D Filters Designed in Polar Coordinates}

We will approach next a particular class of 2D filters, namely filters whose frequency response is symmetric about the origin and has at the same time an angular periodicity. The contour plots of their frequency response, resulted as sections with planes parallel with the frequency plane, can be defined as closed curves which can be described in terms of a variable radius which is a periodic function of the current angle formed with one of the axes. Therefore it can be described in polar coordinates by $\rho=\rho(\varphi)$ where $\varphi$ is the angle formed by the radius OP with $\omega_{1}$-axis, as shown in Fig. 8 (a) for a four-lobe filter. Therefore $\rho(\varphi)$ is a periodic function of the angle $\varphi$ in the range $\varphi \in[0,2 \pi]$.

The proposed design method is based on a zero-phase prototype whose real-valued transfer function can be expressed as a ratio of polynomials in even powers of the frequency $\omega$ :

$$
H_{p}(\omega)=\sum_{j=0}^{M} b_{j} \cdot \omega^{2 j} / \sum_{k=0}^{N} a_{k} \cdot \omega^{2 k}
$$

where $\mathrm{M} \leq \mathrm{N}$ and $\mathrm{N}$ is the filter order. This function may be obtained using a rational approximation of a prototype filter magnitude (e.g. Chebyshev, elliptic). The proposed design method for this class of 2D filters is based on a frequency transformation of the form:

$$
\mathrm{F}: \mathbb{R} \rightarrow \mathbb{C}^{2}, \omega^{2} \rightarrow \mathrm{F}\left(\mathrm{z}_{1}, \mathrm{z}_{2}\right)
$$

The frequency transformation (69) maps the real frequency axis $\omega$ onto the complex plane $\left(\mathrm{z}_{1}, \mathrm{z}_{2}\right)$ and will be defined by a frequency mapping of the form:

$$
\mathrm{F}_{1}: \mathbb{R} \rightarrow \mathbb{R}^{2}, \quad \omega^{2} \rightarrow \mathrm{F}_{1}\left(\omega_{1}, \omega_{2}\right)=\left(\omega_{1}^{2}+\omega_{2}^{2}\right) / \rho\left(\omega_{1}, \omega_{2}\right)
$$

$\rho\left(\omega_{1}, \omega_{2}\right)$ plays the role of a radial compressing function and is initially determined in the angle variable $\varphi$ as $\rho(\varphi)$. In the frequency plane $\left(\omega_{1}, \omega_{2}\right)$ we have:

$$
\cos \varphi=\omega_{1} / \sqrt{\omega_{1}^{2}+\omega_{2}^{2}}
$$


If the radial function $\rho(\varphi)$ can be expressed in the variable $\cos \varphi$, using (71) we obtain by substitution the function $\rho\left(\omega_{1}, \omega_{2}\right)$. We will express the function $\rho(\varphi)$ as a polynomial or a ratio of polynomials in the variable $\cos \varphi$. For instance, the four-lobe filter with contour plot given in Fig.8 (a) corresponds to a function:

$$
\rho(\varphi)=a+b \cos 4 \varphi=a+b-8 b \cos ^{2} \varphi+8 b \cos ^{4} \varphi
$$

plotted in Fig.8 (b) for $\varphi \in[0,2 \pi]$. As 1D prototype we consider a type-2 Chebyshev digital filter with the parameters: order $\mathrm{N}=4$, stopband attenuation $\mathrm{R}_{\mathrm{s}}=40 \mathrm{~dB}$ and passbandedge frequency $\omega_{\mathrm{p}}=0.5$ (1.0 is half the sampling frequency). The transfer function in $z$ is:

$$
\mathrm{H}_{\mathrm{p}}(\mathrm{z})=\left(0.012277 \cdot \mathrm{z}^{2}-0.012525 \cdot \mathrm{z}+0.012277\right) /\left(\mathrm{z}^{2}-1.850147 \cdot \mathrm{z}+0.862316\right)
$$

Its magnitude for $\omega \in[-\pi, \pi]$ is shown in Fig.8 (c). Using the Chebyshev-Padé method and a symbolic computation software, we determine the real-valued transfer function which accurately approximates the magnitude of the digital filter function $\mathrm{H}_{\mathrm{p}}(\mathrm{z})$ :

$$
\mathrm{H}_{\mathrm{a} 1}(\mathrm{~s})=\left(0.9403+0.5756 \cdot \mathrm{s}^{2}+0.0947 \cdot \mathrm{s}^{4}\right) /\left(1+2.067753 \cdot \mathrm{s}^{2}+4.66314 \cdot \mathrm{s}^{4}\right)
$$

This method can be applied for any prototype like (73). More generally, the 2D filter in polar coordinates can be rotated in the frequency plane with a specified angle $\varphi_{0}$ about one of the frequency axes, e.g. $\mathrm{O}-\omega_{2}$. For instance, in the case of a four-lobe filter, two opposite lobes are oriented along a direction at an angle $\varphi_{0}$, and the other two at $\varphi_{0}+\pi / 2$, as in Fig.9 (d). It can be shown that the cosine of the current angle $\varphi$ with initial phase $\varphi_{0}$ can be expressed:

$$
\cos ^{2}\left(\varphi+\varphi_{0}\right)=\left(\cos ^{2} \varphi_{0} \cdot \omega_{1}^{2}+\sin ^{2} \varphi_{0} \cdot \omega_{2}^{2}+0.5 \sin 2 \varphi_{0} \cdot \omega_{1} \omega_{2}\right) /\left(\omega_{1}^{2}+\omega_{2}^{2}\right)
$$

A filter with $\varphi_{0} \neq 0$ is designed in subsection 5.2. For filters with an even number of lobes, as shown further, the radial function $\rho(\varphi)$ is expressed in even powers of $\cos \varphi$ or $\cos \left(\varphi+\varphi_{0}\right)$.

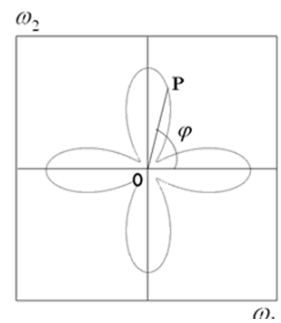

(a)

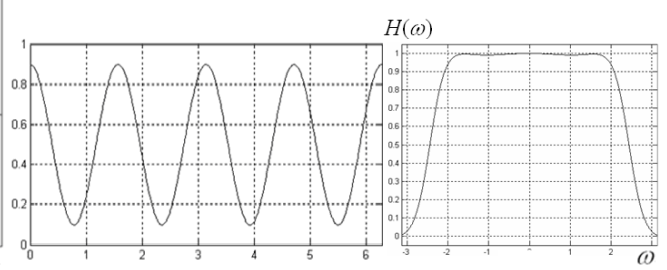

(b)

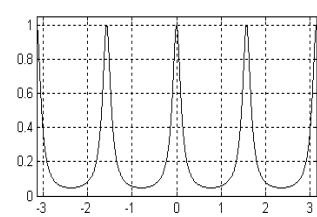

(d)

Fig. 8. (a) Contour plot of a four-lobe filter; (b) variation of the periodic function $\rho(\varphi)$;

(c) maximally-flat low-pass prototype; (d) very selective radial function 
Next we approach the design of several types of recursive zero-phase 2D filters belonging to this class, namely two-lobe and four-lobe filters, fan filters and diamond-shaped filters. The transformation $\omega^{2} \rightarrow \mathrm{F}\left(\mathrm{z}_{1}, \mathrm{z}_{2}\right)$ and the filter frequency response is calculated in each case.

\subsection{Two-Lobe Filter}

A very simple 2D filter belonging to this class is one given by a function $\rho(\varphi)$ of the form:

$$
\rho(\varphi)=a+b \cos 2 \varphi=a-b+2 b \cos ^{2} \varphi
$$

Using (70), (71) and (76) we get the frequency transformation:

$$
\omega^{2} \rightarrow \mathrm{F}_{1}\left(\omega_{1}, \omega_{2}\right)=\left(\omega_{1}^{2}+\omega_{2}^{2}\right)^{2} /\left((\mathrm{a}+\mathrm{b}) \cdot \omega_{1}^{2}+(\mathrm{a}-\mathrm{b}) \cdot \omega_{2}^{2}\right)
$$

Since $\omega_{1}^{2}=-s_{1}^{2}$ and $\omega_{2}^{2}=-s_{2}^{2}$ we get the function $F_{1}\left(s_{1}, s_{2}\right)$ in the complex plane $\left(s_{1}, s_{2}\right)$ :

$$
\mathrm{F}_{1}\left(\mathrm{~s}_{1}, \mathrm{~s}_{2}\right)=-\left(\mathrm{s}_{1}^{2}+\mathrm{s}_{2}^{2}\right)^{2} /\left((\mathrm{a}+\mathrm{b}) \cdot \mathrm{s}_{1}^{2}+(\mathrm{a}-\mathrm{b}) \cdot \mathrm{s}_{2}^{2}\right)
$$

Finally we derive a transfer function of the $2 \mathrm{D}$ filter $\mathrm{H}\left(\mathrm{z}_{1}, \mathrm{z}_{2}\right)$ in the complex plane $\left(\mathrm{z}_{1}, \mathrm{z}_{2}\right)$. This can be achieved if we find a discrete counterpart $R\left(z_{1}, z_{2}\right)$ of the function $\rho\left(\omega_{1}, \omega_{2}\right)$. A possible method is to express the function $\rho\left(\omega_{1}, \omega_{2}\right)$ in the complex plane $\left(s_{1}, s_{2}\right)$ and then find the appropriate mapping to $\left(z_{1}, z_{2}\right)$ using the bilinear transform for the variables $s_{1}$, $s_{2}$. Using (40) in (78), we find the frequency transformation in $z_{1}, z_{2}$ in matrix form:

$$
\omega^{2} \rightarrow \mathrm{F}\left(\mathrm{z}_{1}, \mathrm{z}_{2}\right)=\mathrm{B}\left(\mathrm{z}_{1}, \mathrm{z}_{2}\right) / \mathrm{A}\left(\mathrm{z}_{1}, \mathrm{z}_{2}\right)=\left(\mathbf{Z}_{1} \times \mathbf{B} \times \mathbf{Z}_{2}^{\mathrm{T}}\right) /\left(\mathbf{Z}_{1} \times \mathbf{A} \times \mathbf{Z}_{2}^{\mathrm{T}}\right)
$$

with $\mathbf{Z}_{1}, \mathbf{Z}_{2}$ given in (27). The templates $\mathbf{B}, \mathbf{A}$ giving the coefficients of $\mathrm{B}\left(\mathrm{z}_{1}, \mathrm{z}_{2}\right), \mathrm{A}\left(\mathrm{z}_{1}, \mathrm{z}_{2}\right)$ result as convolutions of $3 \times 3$ matrices: $\mathbf{B}=8 \cdot \mathbf{B}_{1} * \mathbf{B}_{1}, \mathbf{A}=\mathbf{A}_{1} * \mathbf{A}_{2}$, where:

$$
\mathbf{B}_{1}=\left[\begin{array}{rrr}
1 & 0 & 1 \\
0 & -4 & 0 \\
1 & 0 & 1
\end{array}\right] ; \quad \mathbf{A}_{1}=\left[\begin{array}{rrr}
a & 2 b & a \\
-2 b & -4 a & -2 b \\
a & 2 b & a
\end{array}\right] ; \quad \mathbf{A}_{2}=\left[\begin{array}{rrr}
1 & 2 & 1 \\
2 & 4 & 2 \\
1 & 2 & 1
\end{array}\right]
$$

The parameters $\mathrm{a}$ and $\mathrm{b}$ from (76) are chosen imposing the minimum and maximum values of $\rho(\varphi), \mathrm{m}=\mathrm{a}-\mathrm{b}$ and $\mathrm{M}=\mathrm{a}+\mathrm{b}$. For instance with $\mathrm{m}=0.04, \mathrm{M}=4$ we get $\mathrm{a}=2.02$, $\mathrm{b}=1.98$. We next use the maximally-flat filter prototype (74). We substitute the mapping (79) into the general prototype (36) and get the desired 2D transfer function:

$$
H\left(z_{1}, z_{2}\right)=\frac{b_{2} B^{2}\left(z_{1}, z_{2}\right)+b_{1} A\left(z_{1}, z_{2}\right) B\left(z_{1}, z_{2}\right)+b_{0} A^{2}\left(z_{1}, z_{2}\right)}{a_{2} B^{2}\left(z_{1}, z_{2}\right)+a_{1} A\left(z_{1}, z_{2}\right) B\left(z_{1}, z_{2}\right)+A^{2}\left(z_{1}, z_{2}\right)}=B_{f}\left(z_{1}, z_{2}\right) / A_{f}\left(z_{1}, z_{2}\right)
$$


where the coefficients $b_{0}, b_{1}, b_{2}, a_{1}, a_{2}$ may take the values in (74). Since function (81) can be described by the templates $\mathbf{B}_{\mathrm{f}}, \mathbf{A}_{\mathrm{f}}$ corresponding to $\mathrm{B}_{\mathrm{f}}\left(\mathrm{z}_{1}, \mathrm{z}_{2}\right), \mathrm{A}_{\mathrm{f}}\left(\mathrm{z}_{1}, \mathrm{z}_{2}\right)$, we have:

$$
\mathbf{B}_{\mathrm{f}}=\mathrm{b}_{2} \cdot \mathbf{B} * \mathbf{B}+\mathrm{b}_{1} \cdot \mathbf{A} * \mathbf{B}+\mathrm{b}_{0} \cdot \mathbf{A} * \mathbf{A} \quad \mathbf{A}_{\mathrm{f}}=\mathrm{a}_{2} \cdot \mathbf{B} * \mathbf{B}+\mathrm{a}_{1} \cdot \mathbf{A} * \mathbf{B}+\mathbf{A} * \mathbf{A}
$$

where * denotes matrix convolution. For our filter, the templates $\mathbf{B}_{\mathrm{f}}$ and $\mathbf{A}_{\mathrm{f}}$ result of size $9 \times 9$. In Fig. 9 (a) the two-lobe filter frequency response is shown.

\subsection{Very Selective Four-Lobe Filter}

The design of a very selective four-lobe filter in polar coordinates was presented in (Matei, $2009, \mathrm{~b}$ ) and is briefly reconsidered as follows. Let us consider the radial function:

$$
\mathrm{H}_{\mathrm{r}}(\varphi)=1 /(\mathrm{p} \cdot \tilde{\mathrm{B}}(\varphi)-\mathrm{p}+1)
$$

where $\tilde{\mathrm{B}}(\varphi)$ is a periodic function; let $\tilde{\mathrm{B}}(\varphi)=\cos (4 \varphi)$. We use this function to design a $2 \mathrm{D}$ filter with four narrow lobes in the frequency plane. Using trigonometric identities, we get:

$$
\mathrm{H}_{\mathrm{r}}(\varphi)=1 /\left(1+8 \mathrm{p} \cdot(\cos \varphi)^{2}-8 \mathrm{p} \cdot(\cos \varphi)^{4}\right)
$$

plotted for $\varphi \in[-\pi, \pi]$ in Fig.8 (d). This periodic function has the period $\Phi=\pi / 4$ and the shape of a "comb" filter. In order to control the shape of this function, we introduce another parameter $k$, such that the radial function $\rho(\varphi)$ becomes $\rho(\varphi)=k \cdot H_{r}(\varphi)$. We get using (70):

$$
\begin{gathered}
\omega^{2} \rightarrow \mathrm{F}\left(\omega_{1}, \omega_{2}\right)=\left(\omega_{1}^{4}+(2+8 \mathrm{p}) \omega_{1}^{2} \omega_{2}^{2}+\omega_{2}^{4}\right) /\left(\mathrm{k}\left(\omega_{1}^{2}+\omega_{2}^{2}\right)\right) \\
\mathrm{F}_{2}\left(\mathrm{~s}_{1}, \mathrm{~s}_{2}\right)=-\left(\mathrm{s}_{1}^{4}+(2+8 \mathrm{p}) \mathrm{s}_{1}^{2} \mathrm{~s}_{2}^{2}+\mathrm{s}_{2}^{4}\right) /\left(\mathrm{k}\left(\mathrm{s}_{1}^{2}+\mathrm{s}_{2}^{2}\right)\right)
\end{gathered}
$$

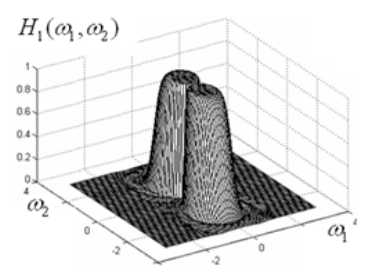

(a)

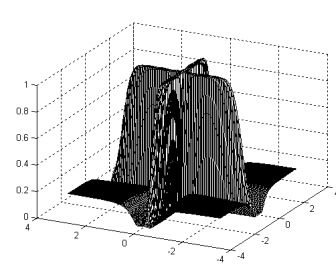

(b)

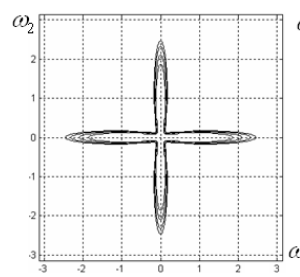

(c)

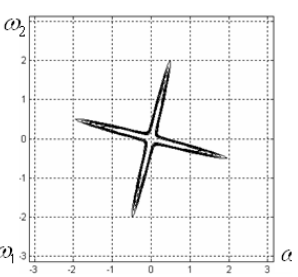

(d)

Fig. 9. (a) Frequency response of the 2-lobe filter; (b), (c) frequency response and contour plot for a narrow 4-lobe filter; (d) contour plot of a rotated 4-lobe filter 
As in the previous example we find the transformation of the same form (79), where $\mathbf{Z}_{1}$ and $\mathbf{Z}_{2}$ are the vectors given by (27), and $\mathbf{B}, \mathbf{A}$ are the $5 \times 5$ matrices:

$$
\mathbf{B}=8 \cdot\left[\begin{array}{ccccc}
-1-2 p & 0 & 4 p-2 & 0 & -1-2 p \\
0 & 8 & 0 & 8 & 0 \\
4 p-2 & 0 & -8 p-20 & 0 & 4 p-2 \\
0 & 8 & 0 & 8 & 0 \\
-1-2 p & 0 & 4 p-2 & 0 & -1-2 p
\end{array}\right] \quad \mathbf{A}=\mathrm{k} \cdot\left[\begin{array}{ccc}
1 & 0 & 1 \\
0 & -4 & 0 \\
1 & 0 & 1
\end{array}\right] *\left[\begin{array}{ccc}
1 & 2 & 1 \\
2 & 4 & 2 \\
1 & 2 & 1
\end{array}\right]=\mathrm{k} \cdot \mathbf{A}_{1} * \mathbf{A}_{2}
$$

Using the prototype (74) we get a transfer function $\mathrm{H}\left(\mathrm{z}_{1}, \mathrm{z}_{2}\right)$ similar to (81) and the templates result from (82). The designed filter has the frequency response and contour plot as in Fig. 9 (b), (c). We remark that the filter is very selective simultaneously along both axes.

The same procedure can be applied to design a four-lobe filter with a specified inclination angle. Using the double bilinear transform (40), the expression (75) for $\cos ^{2}\left(\varphi+\varphi_{0}\right)$ corresponds to the following frequency transformation in the complex variables $z_{1}, z_{2}$ :

$$
\cos ^{2}\left(\varphi+\varphi_{0}\right) \rightarrow \mathrm{F}\left(\mathrm{z}_{1}, \mathrm{z}_{2}\right)=\mathrm{B}\left(\mathrm{z}_{1}, \mathrm{z}_{2}\right) / \mathrm{A}\left(\mathrm{z}_{1}, \mathrm{z}_{2}\right)=\left(\mathbf{Z}_{1} \times \mathbf{B}_{\mathrm{C}} \times \mathbf{Z}_{2}^{\mathrm{T}}\right) /\left(\mathbf{Z}_{1} \times \mathbf{A}_{\mathbf{C}} \times \mathbf{Z}_{2}^{\mathrm{T}}\right)
$$

where $\mathbf{A}_{\mathbf{C}}=2 \cdot \mathbf{A}_{1}$ with $\mathbf{A}_{1}$ given in (87) and

$$
\mathbf{B}_{\mathbf{C}}=\left[\begin{array}{ccc}
1+0.5 \sin \left(2 \varphi_{0}\right) & -2 \cos \left(2 \varphi_{0}\right) & 1-0.5 \sin \left(2 \varphi_{0}\right) \\
2 \cos \left(2 \varphi_{0}\right) & -4 & 2 \cos \left(2 \varphi_{0}\right) \\
1-0.5 \sin \left(2 \varphi_{0}\right) & -2 \cos \left(2 \varphi_{0}\right) & 1+0.5 \sin \left(2 \varphi_{0}\right)
\end{array}\right]
$$

The radial compression function for this filter will be $\rho(\varphi)=k /\left(1+8 p \cdot(\cos \varphi)^{2}-8 p \cdot(\cos \varphi)^{4}\right)$ corresponding to the following pair of $5 \times 5$ matrices:

$$
\mathbf{B}_{\rho}=\mathrm{k} \cdot \mathbf{A}_{\mathrm{C}} * \mathbf{A}_{\mathrm{C}} \quad \mathbf{A}_{\rho}=\mathbf{A}_{\mathrm{C}} * \mathbf{A}_{\mathrm{C}}+8 \mathrm{p} \cdot \mathbf{B}_{\mathrm{C}} * \mathbf{A}_{\mathrm{C}}-8 \mathrm{p} \cdot \mathbf{B}_{\mathrm{C}} * \mathbf{B}_{\mathrm{C}}
$$

The final frequency transformation is given by (79), where $\mathbf{B}=-4 \cdot \mathbf{A}_{\rho}, \mathbf{A}=\mathrm{k} \cdot \mathbf{A}_{\mathrm{C}} * \mathbf{A}_{2}$ and $\mathbf{A}_{2}$ results from (87).

\subsection{Fan Filter Design in Polar Coordinates}

Besides the design method based on wedge filters addressed in subsection 4.3, fan filters can also be designed in polar coordinates. Let us consider the symmetric fan-type filter specified in the plane $\left(\omega_{1}, \omega_{2}\right)$ as in Fig.7 (a), given in the ideal case by relation (64). 
The fan filter contour can be exactly described as:

$$
\rho(\varphi)=\left\{\begin{array}{cc}
\pi / \cos \varphi & \text { for } \varphi \in[-\pi / 4, \pi / 4] \cup[3 \pi / 4,5 \pi / 4] \\
0 & \text { otherwise }
\end{array}\right.
$$

Using a change of variable and a Chebyshev-Padé approximation, we obtain the following approximation $\rho_{\mathrm{a}}(\varphi)$ of $\rho(\varphi)$ for $\varphi \in[-\pi / 2, \pi / 2]$ :

$$
\rho_{\mathrm{a}}(\varphi) \cong 0.1424 \cdot\left(\cos ^{4} \varphi-0.106111 \cos ^{2} \varphi-0.01047\right) /\left(\cos ^{4} \varphi-1.401727 \cos ^{2} \varphi+0.544317\right)
$$

As before, we looked for an expression in $\cos ^{2} \varphi$ in order to substitute the relation (71). We get an expression for $\rho\left(\omega_{1}, \omega_{2}\right)$, then we write it in the plane $\left(\mathrm{s}_{1}, \mathrm{~s}_{2}\right)$ and finally find a frequency transformation similar to (79). The templates $\mathbf{B}$ and $\mathbf{A}$ result of size $5 \times 5$, and $\mathbf{A}$ can be decomposed as a convolution of $3 \times 3$ templates: $\mathbf{A}=\mathbf{A}_{\mathbf{1}} * \mathbf{A}_{\mathbf{1}}$ where $\mathbf{A}_{\mathbf{1}}$ is given in (87). The frequency response of the fan filter preserves the $1 \mathrm{D}$ prototype maximally-flat characteristics in the pass-band.

\subsection{Diamond-Shaped Filters Design in Polar Coordinates}

In this section a new analytical design method for diamond-shaped filters is described, using the above-discussed approach in polar coordinates (Matei, 2010).

As a first step, we determine analytically the mapping which transforms a circle of given radius, in the frequency plane, into a square, having its vertices on the same circle. We refer to the geometrical construction in Fig.10 (a). In the frequency plane $\left(\omega_{1}, \omega_{2}\right)$ spanned by the axes $\mathrm{O} \omega_{1}, \mathrm{O} \omega_{2}$, we consider the circle of radius $\mathrm{R}$. The default value will be $\mathrm{R}=\pi$.

Let us take an arbitrary point $P_{1}$ situated on the first side of the square $\left(A_{1} A_{2}\right)$, and let $\varphi$ be the angle between the segment $\mathrm{OP}_{1}$ and the axis $\mathrm{O} \omega_{1} ; \varphi_{0}$ is the angle between $\mathrm{OA}_{1}$ and axis $\mathrm{O} \omega_{1}$, where $\mathrm{A}_{1}$ is the first vertex of the square. In the triangle $\mathrm{P}_{1} \mathrm{OA}_{1}$ we have the angles: $\prec \mathrm{OA}_{1} \mathrm{P}_{1}=\pi / 4 ; \prec \mathrm{P}_{1} \mathrm{OA}_{1}=\varphi-\varphi_{0} ; \prec \mathrm{OP}_{1} \mathrm{~A}_{1}=3 \pi / 4-\varphi+\varphi_{0}$. Applying the sine theorem in the triangle $\mathrm{P}_{1} \mathrm{OA}_{1}$, we find the measure of segment $\mathrm{OP}_{1}$ as a function of $\mathrm{R}$ and $\varphi$ :

$$
\mathrm{OP}_{1}=\mathrm{R} \cdot \sin \left(\mathrm{OA}_{1} \mathrm{P}_{1}\right) / \sin \left(\mathrm{OP}_{1} \mathrm{~A}_{1}\right)=(\mathrm{R} \sqrt{2} / 2) / \cos \left(\varphi-\varphi_{0}-\pi / 4\right)
$$

Thus we found the measure of $\mathrm{OP}_{1}$ as a function of the current angle. However, (93) is valid only in the range: $\varphi \in\left[\varphi_{0}+2 n \pi / 4, \varphi_{0}+2(n+1) \pi / 4\right]$. For a standard diamond filter $\varphi_{0}=0, R=1$ and in the first quadrant of the frequency plane $\rho(\varphi)=1 / \sqrt{2} \cos (\varphi-\pi / 4)$. To express the value $\mathrm{OP}_{\mathrm{n}}$ for an arbitrary angle $\varphi$, when point $\mathrm{P}_{\mathrm{n}}$ is located on any side of the square, including the vertices, we find a periodic function $\rho(\varphi)$ of the current angle $\varphi$. This function has the period $\Phi=\pi / 2$ and is plotted in Fig.10 (b). A convenient way to obtain a closed-form periodic approximation of this function is by using a rational 
approximation (e.g. Chebyshev-Padé). We look for such an approximation of the function $\rho(\varphi)=1 / \cos \varphi$ for a phase $\varphi \in[-\pi / 4, \pi / 4]$, in powers of the variable $\cos 4 \varphi$, which is a periodic function with period $\pi / 2$. Thus, the rational function will actually approximate the function $\rho(\varphi)$ over the entire range $[0,2 \pi]$. Since $\rho(\varphi)$ is not differentiable in the points $\varphi=-\pi,-\pi / 2,0, \pi / 2$ (corresponding to square vertices), as can be noticed in Fig.10 (b), we consider the function $\rho_{1}(\varphi)$ on the range $\varphi \in[-\pi / 4, \pi / 4]$, which is differentiable everywhere within this interval; we obtain:

$$
\rho(\varphi)=1 / \cos \varphi \cong\left(1+0.087481 \cdot \varphi^{2}\right) /\left(1-0.413 \cdot \varphi^{2}\right)
$$

Now we use the variable change $x=\cos (4 \varphi)$ getting the intermediate function in variable $x$ :

$$
\rho_{\mathrm{i}}(\mathrm{x})=\left(1.082679+1.189232 \cdot \mathrm{x}+0.202714 \cdot \mathrm{x}^{2}\right) /\left(1+1.202559 \cdot \mathrm{x}+0.271879 \cdot \mathrm{x}^{2}\right)
$$

Returning to the initial variable $\varphi=0.25 \cdot \arccos x$, by substituting back $x=\cos (4 \varphi)$, we obtain a rational approximation in powers of $\cos (4 \varphi)$. In this expression we must replace $\varphi$ by $\varphi-\pi / 4$, to get the final approximation for the function $\rho(\varphi)$ :

$$
\rho_{1}(\varphi)=\frac{1.04234-1.046915 \cdot \cos (4 \varphi)+0.089227 \cdot \cos (8 \varphi)}{1-1.058647 \cdot \cos (4 \varphi)+0.119671 \cdot \cos (8 \varphi)} \cong \rho(\varphi)
$$

$\rho_{1}(\varphi)$ is plotted in Fig.10 (c) and is an accurate approximation of the original function $\rho(\varphi)$. Using trigonometric identities, this becomes a rational expression in $(\cos \varphi)^{2 n}$ with $n=1 \ldots 4$.

$$
\rho(x)=0.7456 \cdot \frac{(x+0.347)(x+0.0156)(x-1.0156)(x-1.347)}{(x+0.2342)(x+0.0136)(x-1.0136)(x-1.2342)}
$$

where by $\mathrm{x}$ we denoted here $(\cos \varphi)^{2}$. At this point, substituting $\mathrm{x}=(\cos \varphi)^{2}=\omega_{1}^{2} /\left(\omega_{1}^{2}+\omega_{2}^{2}\right)$ we finally reach an expression of the radial function $\rho(\varphi)$ of the frequency variables $\omega_{1}$ and $\omega_{2}$, i.e. $\rho\left(\omega_{1}, \omega_{2}\right)$.

Next a more general design method for a diamond shaped filter is proposed. It starts from a digital filter prototype, with transfer function $\mathrm{H}(\mathrm{z})$ of order $\mathrm{N}$. We discuss the common case when the numerator and denominator of $\mathrm{H}(\mathrm{z})$ are polynomials in $\mathrm{z}$ of equal degrees. Let us consider a transfer function $\mathrm{H}(\mathrm{z})$ of even order $\mathrm{N}$, factorized into second order functions (biquads), with the general form (3) and the frequency response (5), defined in section 2. In the case of diamond filters, the frequency mapping defined in (70) is modified, becoming:

$$
\mathrm{F}_{1}: \mathbb{R} \rightarrow \mathbb{R}^{2}, \omega \rightarrow \mathrm{F}_{1}\left(\omega_{1}, \omega_{2}\right)=\sqrt{\omega_{1}^{2}+\omega_{2}^{2}} / \rho\left(\omega_{1}, \omega_{2}\right)
$$




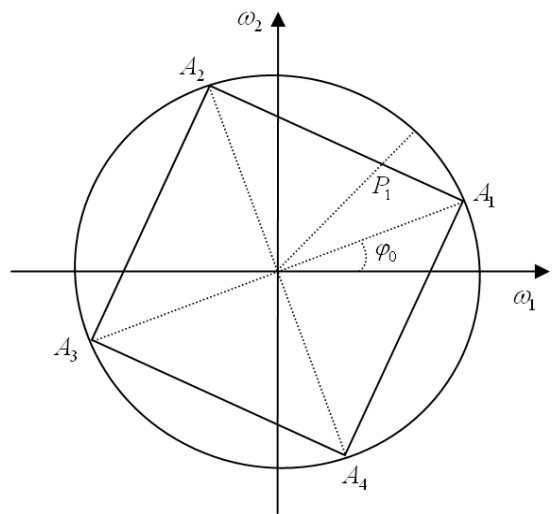

(a)

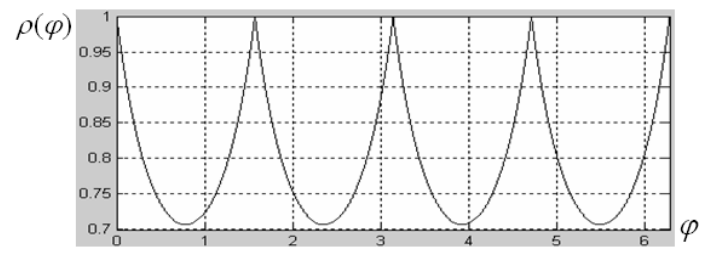

(b)

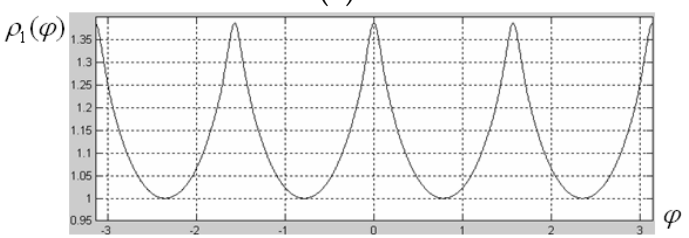

(c)

Fig. 10. (a) Square inscribed in the circle of radius $R$ in the frequency plane, with an initial phase $\varphi_{0} ;(b)$ periodic function $\rho(\varphi) ;(b)$ its periodic approximation $\rho_{1}(\varphi)$

The expression (96), using trigonometric identities, can be written in powers of $(\cos \varphi)^{2}$; then, according to (71) we have $(\cos \varphi)^{2}=\omega_{1}^{2} /\left(\omega_{1}^{2}+\omega_{2}^{2}\right)$ and by substitution we obtain an expression of the radial function $\rho(\varphi)$ in the two frequency variables $\omega_{1}$ and $\omega_{2}$, denoted $\rho\left(\omega_{1}, \omega_{2}\right)$. Finally we get an expression of the real frequency transformation of the general form (98). The next step is to find numerically approximations of the functions:

$$
\mathrm{C}\left(\omega_{1}, \omega_{2}\right)=\cos \left(\sqrt{\omega_{1}^{2}+\omega_{2}^{2}} / \rho\left(\omega_{1}, \omega_{2}\right)\right), \mathrm{S}\left(\omega_{1}, \omega_{2}\right)=\sin \left(\sqrt{\omega_{1}^{2}+\omega_{2}^{2}} / \rho\left(\omega_{1}, \omega_{2}\right)\right)
$$

We will approximate the above functions using a trigonometric series of the general form:

$$
F\left(\omega_{1}, \omega_{2}\right) \cong \sum_{m=-N}^{N} \sum_{n=-N}^{N} a_{m n} \cos \left(m \omega_{1}+n \omega_{2}\right)
$$

where $\mathrm{N}$ is imposed by the required precision. This approximation is derived indirectly, using again the change of variables: $\omega_{1}=\arccos x_{1}, \omega_{2}=\arccos x_{2}$. Thus we obtain from $C\left(\omega_{1}, \omega_{2}\right)$ and $S\left(\omega_{1}, \omega_{2}\right)$ the functions $C_{x}\left(x_{1}, x_{2}\right)$ and $S_{x}\left(x_{1}, x_{2}\right)$ with rather complicated expressions. However, using a symbolic calculation software, we can derive immediately the bivariate Taylor series expansion in $x_{1}$ and $x_{2}$, of the general form:

$$
\mathrm{F}_{\mathrm{x}}\left(\mathrm{x}_{1}, \mathrm{x}_{2}\right) \cong \sum_{\mathrm{k}=-\mathrm{N}}^{\mathrm{N}} \sum_{\mathrm{l}=-\mathrm{N}}^{\mathrm{N}} \mathrm{b}_{\mathrm{kl}} \cdot \mathrm{x}_{1}^{\mathrm{k}} \mathrm{x}_{2}^{1}
$$


Finally by substituting back in (101) $x_{1}=\cos \omega_{1}$ and $x_{2}=\cos \omega_{2}$ we return to the former variables and applying again trigonometric identities we obtain the desired expansions of the form (100). For instance with $N=2$ the expansions for $C\left(\omega_{1}, \omega_{2}\right)$ and $S\left(\omega_{1}, \omega_{2}\right)$ are:

$$
\begin{aligned}
& \mathrm{C}\left(\omega_{1}, \omega_{2}\right) \cong-0.419822+0.517714 \cdot\left(\cos \omega_{1}+\cos \omega_{2}\right)+0.177207 \cdot\left(\cos \left(\omega_{1}+\omega_{2}\right)+\cos \left(\omega_{1}-\omega_{2}\right)\right) \\
& \quad-0.054476 \cdot\left(\cos \left(\omega_{1}+2 \omega_{2}\right)+\cos \left(\omega_{1}-2 \omega_{2}\right)+\cos \left(2 \omega_{1}+\omega_{2}\right)+\cos \left(2 \omega_{1}-\omega_{2}\right)\right) \\
& \quad+0.094109 \cdot\left(\cos 2 \omega_{1}+\cos 2 \omega_{2}\right)-0.008439 \cdot\left(\cos \left(2 \omega_{1}+2 \omega_{2}\right)+\cos \left(2 \omega_{1}-2 \omega_{2}\right)\right) \\
& \mathrm{S}\left(\omega_{1}, \omega_{2}\right) \cong 0.552617+0.393861 \cdot\left(\cos \omega_{1}+\cos \omega_{2}\right)-0.233406 \cdot\left(\cos \left(\omega_{1}+\omega_{2}\right)+\cos \left(\omega_{1}-\omega_{2}\right)\right) \\
& -0.041057 \cdot\left(\cos \left(\omega_{1}+2 \omega_{2}\right)+\cos \left(\omega_{1}-2 \omega_{2}\right)+\cos \left(2 \omega_{1}+\omega_{2}\right)+\cos \left(2 \omega_{1}-\omega_{2}\right)\right) \\
& -0.1238 \cdot\left(\cos 2 \omega_{1}+\cos 2 \omega_{2}\right)+0.009519 \cdot\left(\cos \left(2 \omega_{1}+2 \omega_{2}\right)+\cos \left(2 \omega_{1}-2 \omega_{2}\right)\right)
\end{aligned}
$$

Next, expressing each cosine term as a function of the complex variables $z_{1}=e^{j \omega_{1}}, z_{2}=e^{j \omega_{2}}$ : $\cos \left(m \omega_{1}+n \omega_{2}\right)=0.5\left(z_{1}^{m} z_{2}^{n}+z_{1}^{-m} z_{2}^{-n}\right)$, we get according to (99) the real functions $C_{Z}\left(z_{1}, z_{2}\right)$, $S_{Z}\left(z_{1}, z_{2}\right)$. Through the real frequency transformation (98) we finally reached the mappings:

$$
\cos \omega \rightarrow \mathrm{C}_{\mathrm{Z}}\left(\mathrm{z}_{1}, \mathrm{z}_{2}\right) \quad \sin \omega \rightarrow \mathrm{S}_{\mathrm{Z}}\left(\mathrm{z}_{1}, \mathrm{z}_{2}\right)
$$

Taking into account the expression (5), the 1D biquad function $\mathrm{H}_{2}(\mathrm{z})$ given in (3) is mapped into the following $2 \mathrm{D}$ function $\mathrm{H}_{2 \mathrm{D}}\left(\mathrm{z}_{1}, \mathrm{z}_{2}\right)$ in the variables $\mathrm{z}_{1}$ and $\mathrm{z}_{2}$ :

$$
\mathrm{H}_{2 \mathrm{D}}\left(\mathrm{z}_{1}, \mathrm{z}_{2}\right)=\mathrm{B}\left(\mathrm{z}_{1}, \mathrm{z}_{2}\right) / \mathrm{A}\left(\mathrm{z}_{1}, \mathrm{z}_{2}\right)=\frac{\mathrm{b}_{1}+\left(\mathrm{b}_{0}+\mathrm{b}_{2}\right) \cdot \mathrm{C}_{\mathrm{Z}}\left(\mathrm{z}_{1}, \mathrm{z}_{2}\right)+\mathrm{j} \cdot\left(\mathrm{b}_{2}-\mathrm{b}_{0}\right) \cdot \mathrm{S}_{\mathrm{Z}}\left(\mathrm{z}_{1}, \mathrm{z}_{2}\right)}{\mathrm{a}_{1}+\left(1+\mathrm{a}_{0}\right) \cdot \mathrm{C}_{\mathrm{Z}}\left(\mathrm{z}_{1}, \mathrm{z}_{2}\right)+\mathrm{j} \cdot\left(1-\mathrm{a}_{0}\right) \cdot \mathrm{S}_{\mathrm{Z}}\left(\mathrm{z}_{1}, \mathrm{z}_{2}\right)}
$$

We remark that the obtained 2D filter function has complex coefficients if it is expressed in the $2 \mathrm{D} \mathrm{Z}$ transform. The real functions $\mathrm{C}_{\mathrm{Z}}\left(\mathrm{z}_{1}, \mathrm{z}_{2}\right), \mathrm{S}_{\mathrm{Z}}\left(\mathrm{z}_{1}, \mathrm{z}_{2}\right)$ can further be written as:

$$
\mathrm{C}_{\mathrm{Z}}\left(\mathrm{z}_{1}, \mathrm{z}_{2}\right)=\mathbf{Z}_{1} \times \mathbf{C} \times \mathbf{Z}_{2}^{\mathrm{T}} \quad \mathrm{S}_{\mathrm{Z}}\left(\mathrm{z}_{1}, \mathrm{z}_{2}\right)=\mathbf{Z}_{1} \times \mathbf{S} \times \mathbf{Z}_{2}^{\mathrm{T}}
$$

where the vectors $\mathbf{Z}_{1}, \mathbf{Z}_{2}$ are again given in (27) and $\mathbf{C}, \mathbf{S}$ are matrices of size $5 \times 5$ which have as elements the coefficients identified from the expressions (102) and (103) of $C\left(\omega_{1}, \omega_{2}\right)$ and $S\left(\omega_{1}, \omega_{2}\right)$. For instance the matrix $\mathbf{C}$ results as:

$$
\mathbf{C}=\left[\begin{array}{rrrrr}
0.0471 & -0.0272 & -0.0042 & -0.0272 & 0.0471 \\
-0.0272 & 0.0886 & 0.2588 & 0.0886 & -0.0272 \\
-0.0042 & 0.2588 & -0.4198 & 0.2588 & -0.0042 \\
-0.0272 & 0.0886 & 0.2588 & 0.0886 & -0.0272 \\
0.0471 & -0.0272 & -0.0042 & -0.0272 & 0.0471
\end{array}\right]
$$

where the elements were limited to 4 decimals. The matrices $\mathbf{C}$ and $\mathbf{S}$ have horizontal and vertical symmetry. Since the element values decrease rapidly towards margins, the size 
$5 \times 5$ for the templates $\mathbf{C}$ and $\mathbf{S}$ is sufficient to ensure the accuracy of the numerical approximation, and higher order terms can be ignored with a negligible error. Taking into account relations (105) and (106), we finally express the complex matrices $\mathbf{B}$ and $\mathbf{A}$ that correspond to the numerator and denominator of $\mathrm{H}_{2 \mathrm{D}}\left(\mathrm{z}_{1}, \mathrm{z}_{2}\right)$, i.e. $\mathrm{B}\left(\mathrm{z}_{1}, \mathrm{z}_{2}\right)$ and $\mathrm{A}\left(\mathrm{z}_{1}, \mathrm{z}_{2}\right)$ :

$$
\mathbf{B}=b_{1} \cdot \mathbf{E}+\left(b_{0}+b_{2}\right) \cdot \mathbf{C}+j\left(b_{2}-b_{0}\right) \cdot \mathbf{S} \quad \mathbf{A}=a_{1} \cdot \mathbf{E}+\left(1+a_{0}\right) \cdot \mathbf{C}+j\left(1-a_{0}\right) \cdot \mathbf{S}
$$

By $\mathbf{E}$ we denoted the $5 \times 5$ zero matrix with the central element of value 1 . The mapping of the biquad function $\mathrm{H}_{\mathrm{b}}(\mathrm{z})$ to $\mathrm{H}_{2 \mathrm{D}}\left(\mathrm{z}_{1}, \mathrm{z}_{2}\right)$ can be written as:

$$
\mathrm{H}_{\mathrm{b}}(\mathrm{z}) \rightarrow \mathrm{H}_{2 \mathrm{D}}\left(\mathrm{z}_{1}, \mathrm{z}_{2}\right)=\left(\mathbf{Z}_{1} \times \mathbf{B} \times \mathbf{Z}_{2}^{\mathrm{T}}\right) /\left(\mathbf{Z}_{1} \times \mathbf{A} \times \mathbf{Z}_{2}^{\mathrm{T}}\right)
$$

The filter templates result complex due to the fact that $C\left(\omega_{1}, \omega_{2}\right)$ and $S\left(\omega_{1}, \omega_{2}\right)$ have even parity in $\omega_{1}$ and $\omega_{2}$ and thus can be developed in a trigonometric series of $\cos \left(m \omega_{1}+n \omega_{2}\right)$. Design example. Let us consider the elliptic low-pass prototype filter function

$$
\mathrm{H}(\mathrm{z})=\frac{0.1539 \cdot \mathrm{z}^{4}+0.482 \cdot \mathrm{z}^{3}+0.6734 \cdot \mathrm{z}^{2}+0.482 \cdot \mathrm{z}+0.1539}{\mathrm{z}^{4}+0.155 \cdot \mathrm{z}^{3}+0.7649 \cdot \mathrm{z}^{2}-0.0376 \cdot \mathrm{z}+0.079}
$$

of order $\mathrm{N}=4, \mathrm{R}_{\mathrm{p}}=0.7 \mathrm{~dB}$ passband ripple, a minimum stop-band attenuation $\mathrm{R}_{\mathrm{S}}=40 \mathrm{~dB}$, pass-band edge frequency $\omega_{\mathrm{S}}=0.5$, having a maximally-flat frequency response magnitude, with a relatively steep descent (Fig.11(a)). We design a diamond shaped filter starting from this prototype. $\mathrm{H}(\mathrm{z})$ can be factorized as follows:

$$
H(z)=0.1539 \cdot \frac{\left(z^{2}+1.2884 z+1\right)}{\left(z^{2}+0.2554 z+0.6732\right)} \cdot \frac{\left(z^{2}+1.8425 z+1\right)}{\left(z^{2}-0.1004 z+0.1173\right)}
$$

For the first biquad from (111), we identify the coefficients of the general form (3): $b_{2}=1$, $\mathrm{b}_{1}=1.2884, \mathrm{~b}_{0}=1, \mathrm{a}_{1}=0.2554, \mathrm{a}_{0}=0.6732$. Since $\mathrm{b}_{0}=\mathrm{b}_{2}$, the matrix $\mathbf{B}$ from (108) results real (the imaginary part is cancelled), while matrix A results complex:

$$
\mathbf{B}_{1}=1.2884 \cdot \mathbf{E}+2 \cdot \mathbf{C} \quad \mathbf{A}_{1}=0.2554 \cdot \mathbf{E}+1.6732 \cdot \mathbf{C}+0.3268 \mathrm{j} \cdot \mathbf{S}
$$

For the second biquad from (111) we get as well:

$$
\mathbf{B}_{2}=1.8425 \cdot \mathbf{E}+2 \cdot \mathbf{C} \quad \mathbf{A}_{2}=-0.1004 \cdot \mathbf{E}+1.1173 \cdot \mathbf{C}+0.8827 \mathbf{j} \cdot \mathbf{S}
$$

The final filter templates $\mathbf{B}, \mathbf{A}$ result as convolutions of the templates for the two biquads:

$$
\mathbf{B}=0.1359 \cdot \mathbf{B}_{1} * \mathbf{B}_{2} \quad \mathbf{A}=\mathbf{A}_{1} * \mathbf{A}_{2}
$$

The coefficient in front of $\mathrm{H}(\mathrm{z})$ from (111) was included in $\mathbf{B}$. 


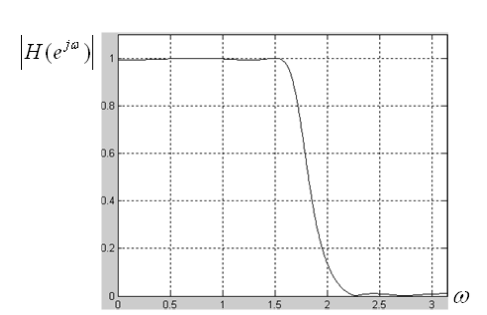

(a)

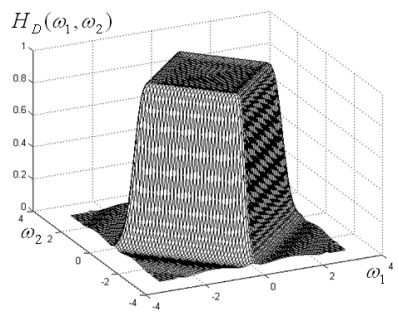

(b)

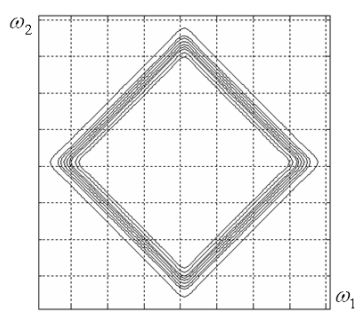

(c)

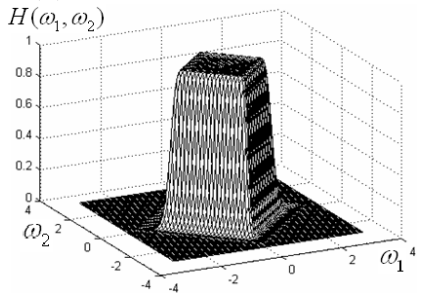

(d)

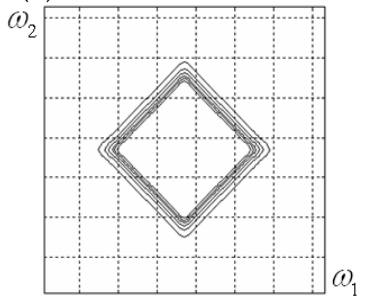

(e)

Fig. 11. (a) Magnitude of the elliptic low-pass prototype filter; frequency responses (b), (d) and contour plots (c), (e) for two diamond filters

\section{Applications and Simulation Results}

All the filters discussed in this chapter have interesting applications in image processing. For the directional filters designed in section 3 some examples are given in (Matei \& Matei, 2009) and for zero-phase directional filters in (Matei, 2009, b).

The wedge filter can be used in image filtering to select from a given image the lines with a specified orientation. The spectrum of a straight line is oriented in the plane $\left(\omega_{1}, \omega_{2}\right)$ at an angle of $\pi / 2$ with respect to the line direction. The binary test image in Fig.12 (a) contains straight lines with different lengths and orientations and is filtered with a maximally-flat wedge filter with aperture $\theta=\pi / 6$ and orientation $\varphi=\pi / 5$, designed using the method from sub-section 4.1. In the filtered image (Fig.12 (b)) only the lines which have the spectrum oriented more or less along the filter characteristic, remain practically unchanged, while all the other lines appear more or less blurred, due to directional low-pass filtering. The directional resolution depends on the filter angular selectivity given by $\theta$. In the second example shown in Fig.12 (c) we consider a real grayscale image representing a straw texture. The straws have random directions and choosing different filter orientations we can select the ones with roughly the same orientation and filter out the rest. The aperture angle was $\theta=\pi / 5$ and three different orientations were used $(\varphi=\pi / 6, \varphi=\pi / 3, \varphi=2 \pi / 3)$, obtaining the filtered images (d), (e), (f). These simple examples illustrate the wedge filter capabilities. 


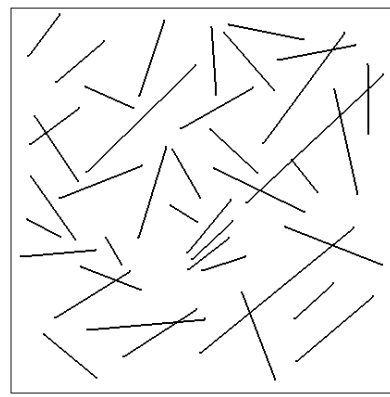

(a)

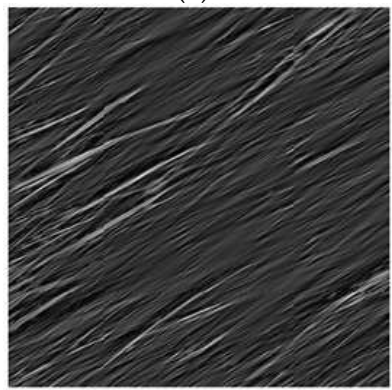

(d)

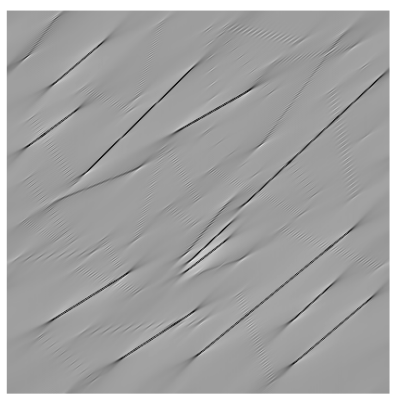

(b)

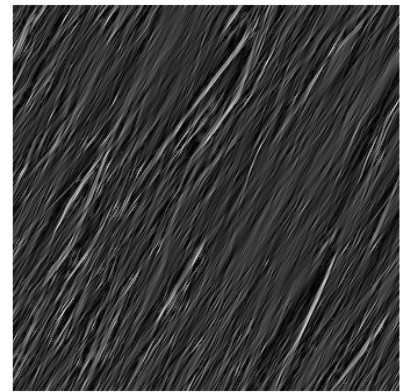

(e)

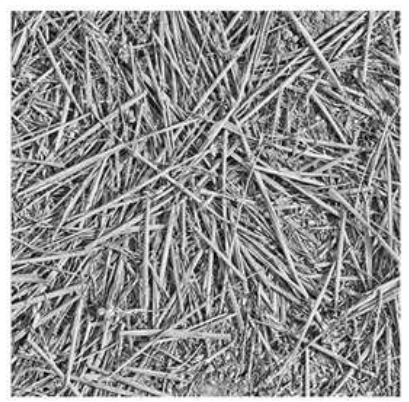

(c)

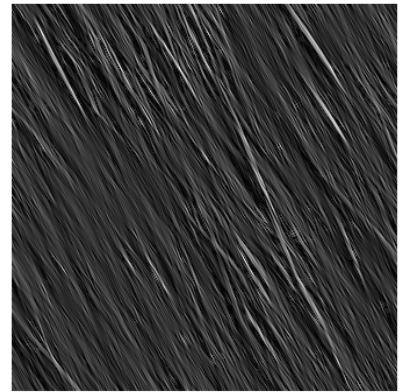

(f)

Fig. 12. (a) Binary test image; (b) wedge filter output $(\theta=\pi / 6, \varphi=\pi / 5)$; (c) grayscale straw texture image; (d), (e), (f) filtering results using $\theta=\pi / 5$ and $\varphi=\pi / 6, \varphi=\pi / 3, \varphi=2 \pi / 3$

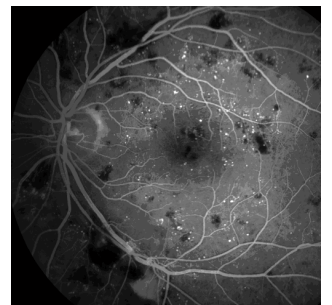

(a)

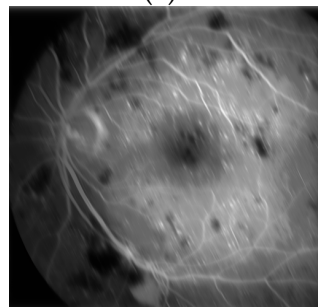

(e)

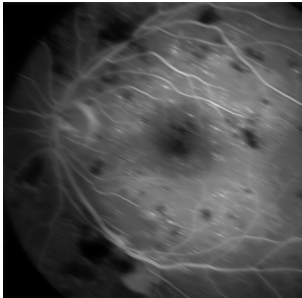

(b)

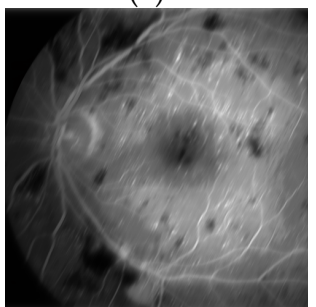

(f)

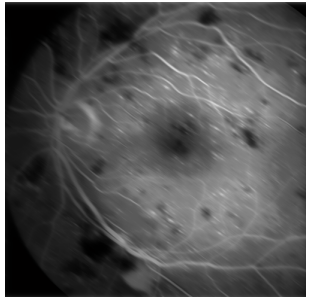

(c)

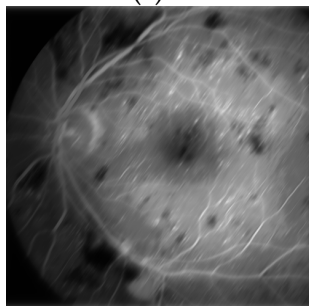

(g)

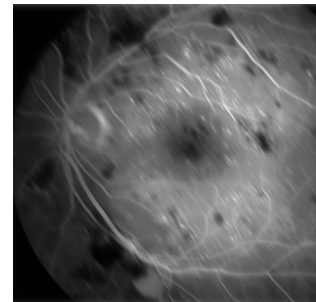

(d)

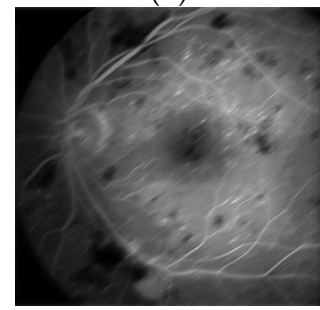

(h)

Fig. 13. (a) Retina angiography; (b)-(h) images resulted as output of the filter bank channels 
Applying the design method for wedge filters with arbitrary aperture and orientation, it is easy to obtain the components of the Bamberger-type filter bank with 8 bands (Fig.5). It is sufficient to design only two adjacent component filters of the bank (bands 5 and 6), the others resulting from symmetry. This filter bank was applied in filtering a typical medical image. The most currently used vascular imaging technique is X-ray angiography, mainly in diagnosing cardio-vascular pathologies, but also in assessing diabetic retinopathy, a severe complication seriously impairing vision. Clinicians usually search in angiograms relevant features like number and position of vessels (arteries, capillaries).

A filter bank like the one presented above may be used in analyzing angiography images by detecting vessels with a given orientation. Let us consider the retina angiogram in Fig.13 (a), featuring some pathological elements indicating a diabetic retinopathy. This image is applied to the designed 8-band wedge filter bank. Fig.13 (b)-(h) show the directionally filtered images. The vessels whose spectrum overlaps more or less with the filter characteristic remain visible, while the others are blurred, an effect of the low-pass filtering.

\section{Conclusion}

The design methods presented in this chapter are mainly analytical but include as well some numerical optimization techniques. The 2D filters result from specified $1 \mathrm{D}$ prototypes with a desired characteristic, usually low-pass and maximally-flat or very selective. Then for each type of 2D filter, a particular spectral transformation is derived. Thus the 2D filter results from its factorized prototype function by a simple substitution. Only recursive filters were approached, since we envisaged obtaining efficient, low-order filters. The designed filters are versatile in the sense that prototype parameters (band-width, selectivity) can be adjusted and the 2D filter will inherit these properties. An advantage of the analytical approach over the completely numerical optimization techniques is the possibility to control the 2D filter parameters by adjusting the prototype. Several types of 2D filters were approached. A novelty is the analytical design method in polar coordinates, which can yield selective twodirectional and even multi-directional filters, and also fan and diamond filters. In polar coordinates more general filters with a specified rotation angle can be synthesized.

Another is the design of zero-phase 2D filters from prototypes with real transfer functions, derived by approximating the magnitude of a common IIR filter. Stability of the designed filters is also an important problem and will be studied in detail in future work on this topic. In principle the spectral transformations used preserve the stability of the 1D prototype. The derived 2D filter could become unstable only if the numerical approximations introduce large errors. In this case the precision of approximation has to be increased by considering higher order terms, which would increase in turn the filter complexity; however, this is the price paid for obtaining efficient and stable 2D filters. Further research will focus on an efficient implementation of the designed filters and also on their applications in real-life image processing.

\section{Acknowledgment}

This work was supported by the National University Research Council under Grant PN2 ID_310 "Algorithms and parallel architectures for signal acquisition, compression and processing". 


\section{References}

Bamberger, R.H. \& Smith, M. A filter bank for the directional decomposition of images: theory and design, IEEE Trans. Signal Processing, Vol. 40(4), Apr. 1992, pp.882-893

Chang, H. \& Aggarwal, J. (1977). Design of two-dimensional recursive filters by interpolation. IEEE Trans. Circuits Systems, vol. CAS-24, pp.281-291, June 1977

Danielsson, P.E. (1980). Rotation-Invariant Linear Operators with Directional Response. Proceedings of 5th International Conf. on Pattern Recognition, Miami, USA, Dec. 1980

Freeman, W.T. \& Adelson, E.H. (1991). The design and use of steerable filters. IEEE Trans. on Pattern Analysis and Machine Intelligence, Vol.13 (9), Sept. 1991, pp.891-906

Harn, L. \& Shenoi, B. (1986). Design of stable two-dimensional IIR filters using digital spectral transformations. IEEE Trans. Circ. Systems, CAS-33, May 1986, pp. 483-490

Hirano, K. \& Aggarwal, J.K. (1978). Design of two-dimensional recursive digital filters. IEEE Trans. Circuits Systems, CAS-25, Dec. 1978, pp.1066-1076

Jury, E.I.; Kolavennu, V.R. \& Anderson, B.D. (1977). Stabilization of certain two-dimensional recursive digital filters. Proceedings of the IEEE, vol. 65, no. 6, 1977, pp. 887-892

Kayran, A. \& King, R. (1983). Design of recursive and nonrecursive fan filters with complex transformations, IEEE Trans. on Circuits and Systems, CAS-30(12), 1983, pp.849-857

Lakshmanan, V. (2004). A separable filter for directional smoothing. IEEE Geoscience and Remote Sensing Letters, July 2004, Vol.1, pp.192-195

Lim, J.S. (1990). Two-Dimensional Signal and Image Processing. Prentice-Hall 1990

Lim, Y.C. \& Low, S.H. (1997). The synthesis of sharp diamond-shaped filters using the frequency response masking approach. Proc. of IEEE Int. Conf. on Acoustics, Speech $\mathcal{E}$ Signal Processing, ICASSP-97, pp.2181-2184, Munich, Germany, Apr. 21-24, 1997

Low, S.H. \& Lim, Y. C. (1998). A new approach to design sharp diamond-shaped filters. Signal Processing, Vol. 67 (1), May 1998, pp. 35-48, ISSN:0165-1684

Lu, W.S. \& Antoniou, A. (1992). Two-Dimensional Digital Filters, CRC Press, 1992

Matei, R. \& Matei, D. (2009). Orientation-selective 2D recursive filter design based on frequency transformations, Proceedings of IEEE Region 8 EUROCON 2009 Conference, pp. 1320-1327, ISBN 978-1-4244-3861-7, St. Petersburg, Russia, May 18-23, 2009

Matei, R. (2009, a). Design Method for Wedge-Shaped Filters, Proceedings of the International Conference on Signal Processing and Multimedia Applications SIGMAP 2009, pp. 1923, ISBN 978-989-674-005-4, Milano, Italy, July 7-10, 2009

Matei, R. (2009, b). New Model and Applications of Cellular Neural Networks in Image Processing, In: “Advanced Technologies", Kankesu Jayanthakumaran (Ed.), pp. 471-501, IN-TECH Vienna, 2009, ISBN: 978-953-307-009-4

Matei, R. (2010). A New Design Method for IIR Diamond-Shaped Filters, Proceedings of the 18th European Signal Processing Conference EUSIPCO 2010, pp. 65-69, ISSN 2076-1465, Aalborg, Denmark, Aug. 23-27, 2010

O'Connor, B.T. \& Huang, T.S. (1978). Stability of general two-dimensional recursive digital filters," IEEE Trans. Acoustics, Speech E Signal Processing, vol.26 (6), 1978, pp.550-560

Pendergrass, N.; Mitra, S.K. \& Jury, E.I. (1976). Spectral transformations for two-dimensional digital filters. IEEE Transactions Circuits \& Systems, vol. CAS-23, Jan. 1976, pp. 26-35

Randen, R. \& Husoy, J.H. (1999). Filtering for texture classification: a comparative study. IEEE Trans. Pattern Analysis and Machine Intelligence, Vol.21(4), Apr.1999, pp.291-310

Simoncelli, E.P. \& Farid, H. (1996). Steerable wedge filters for local orientation analysis. IEEE Trans.on Image Processing, Vol. 5 (9), Sep 1996, pp.1377-1382, ISSN: 1057-7149 
www.intechopen.com 


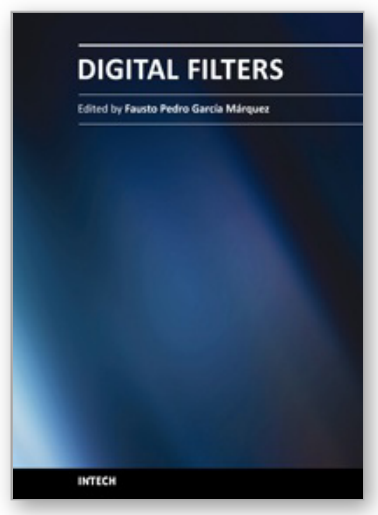

\author{
Digital Filters \\ Edited by Prof. Fausto Pedro GarcÃa MÃ $i r q u e z$
}

ISBN 978-953-307-190-9

Hard cover, 290 pages

Publisher InTech

Published online 11, April, 2011

Published in print edition April, 2011

The new technology advances provide that a great number of system signals can be easily measured with a low cost. The main problem is that usually only a fraction of the signal is useful for different purposes, for example maintenance, DVD-recorders, computers, electric/electronic circuits, econometric, optimization, etc. Digital filters are the most versatile, practical and effective methods for extracting the information necessary from the signal. They can be dynamic, so they can be automatically or manually adjusted to the external and internal conditions. Presented in this book are the most advanced digital filters including different case studies and the most relevant literature.

\title{
How to reference
}

In order to correctly reference this scholarly work, feel free to copy and paste the following:

Radu Matei (2011). New Design Methods for Two-Dimensional Filters Based on 1D Prototypes and Spectral Transformations, Digital Filters, Prof. Fausto Pedro GarcÃa MÃ irquez (Ed.), ISBN: 978-953-307-190-9, InTech, Available from: http://www.intechopen.com/books/digital-filters/new-design-methods-for-twodimensional-filters-based-on-1d-prototypes-and-spectral-transformations

\section{INTECH}

open science | open minds

\section{InTech Europe}

University Campus STeP Ri Slavka Krautzeka 83/A 51000 Rijeka, Croatia

Phone: +385 (51) 770447

Fax: +385 (51) 686166 www.intechopen.com

\section{InTech China}

Unit 405, Office Block, Hotel Equatorial Shanghai No.65, Yan An Road (West), Shanghai, 200040, China 中国上海市延安西路65号上海国际贵都大饭店办公楼405单元 Phone: +86-21-62489820

Fax: $+86-21-62489821$ 
(C) 2011 The Author(s). Licensee IntechOpen. This chapter is distributed under the terms of the Creative Commons Attribution-NonCommercialShareAlike-3.0 License, which permits use, distribution and reproduction for non-commercial purposes, provided the original is properly cited and derivative works building on this content are distributed under the same license. 